\title{
Semantic Algorithm for Industrial Symbiosis Network Synthesis
}

\author{
Franjo Cecelja (corresponding author - email: f.cecelja@surrey.ac.uk, tel/fax: +44 1483 \\ 686 585), Nikolaos Trokanas, Tara Raafat, Mingyen Yu
}

Centre for Process \& Information Systems Engineering, Faculty of Engineering \& Physical Sciences, University of Surrey, Guildford, GU2 7XH, United Kingdom

\begin{abstract}
The paper introduces a semantic algorithm for building Industrial Symbiosis networks. Built around ontology modelling of knowledge in the domain of Industrial Symbiosis, the algorithm enables the acquisition of the explicit knowledge from the user through ontology instantiation and input/output matching based on semantic relevance between the participants. Formation of innovative Industrial Symbiosis networks is enabled by decomposition of properties characterising respective resources and solutions, the process optimised for set environmental criteria. The proposed algorithm is implemented as a web service. The potential of the algorithm is demonstrated by several case studies using real-life data.
\end{abstract}

\section{Keywords}

Industrial symbiosis, Ontology, Semantic matching, Optimisation, Network Synthesis

\section{Introduction}

Based on the principle of industrial ecology to reduce the use of virgin materials and energy by reusing water, recovering energy and utilising by-products, the items commonly called waste, Industrial Symbiosis (IS) describes the industrial networks set on an ad-hoc principle. Such networks focus on trading material, energy and water to gain economic, environmental and social benefits (Lehtoranta et al. 2011). Ad-hoc principle refers to collaboration between companies which normally do not have established consumer/supplier relationship and which occurs within strict geographical and environmental boundaries (Chertow 2004). Economic benefits are generated by the cost efficiency coming from the off-market prices of waste material and energy generation and they are driving force for private industry to participate. Tighter integration enables further economic savings through cascading of water and energy and sharing utilities and services and hence yielding collective benefits greater than the sum of individual benefits (JaeYeon et al. 2006). Tighter integration is also justified by environmental and social grounds. By focusing on reuse of waste, energy and water, environmental benefits are integral part of IS, which include landfill and pollutant savings, reduction in greenhouse gas generations (Mirata and Emtairah 2005), improved resource use efficiency (Chertow 2007) and reduced use of non-renewable resources (Trokanas et al. 2013). These benefits are further amplified by geographical boundaries and localised operation. Some authors claim that localised operation of IS provides measurable outputs in revitalising urban and rural sites, promotes job growth and retention and encourages more sustainable development (Chertow and Ehrenfeld 2012). It has been proven that environmental and social benefits are driving force behind the interest of city planners (Chertow 2004), economic development experts and real estate developers and agencies to take proactive role and to participate and promote (Alberta and Kevin 2008).

In practice, IS occurs locally or regionally as spontaneous process or promoted and otherwise supported by states or regions. Key to establishing symbiosis is the matching of inputs and outputs to make links across industries (Chertow 2004). In contrast to virgin materials, waste materials and waste energy are typically 
nonstandard and off-spec, not originally designed for reuse and highly variable in composition and pattern of availability. This heterogeneity is difficult to define distinctively and inputs and outputs are characterised more by tacit knowledge based on associations, know-how expertise and engineering intuition (Cecelja, F. et al. 2014b). Although rarely measured explicitly in practice, environmental and social benefits are presumed in the process of establishing links which inevitably increases number of options to consider, especially at the early stage of symbiosis. Nonstandard and nonmarket transactions between symbiotic partners also add to the complexity. It is for these reasons that, as at present, the IS is usually initiated and the whole process coordinated manually by trained IS practitioners which normally take active part in the process of decision making; the process which tends to be slow, expensive and coloured by practitioner's experience and expertise.

Realising the complexity of the task and cognitive limitations of practitioners in perplexing situations, purpose built information systems have been introduced to support and facilitate the process. Existing IS support systems are thoroughly reviewed by (Grant et al. 2010). They typically involve opportunity identification by mimicking input-output matching based on explicit data arranged in proprietary databases, with some exceptions which address collaborative projects and workflow management. These support systems are dominantly helpful in the second phase of symbiosis, the phase of operation and monitoring. Such an approach is justifiable by the fact that, with explicit knowledge available, opportunity identification appears to be a logical starting point. Input/output (I/O) matching appears as a simple optimisation routine until more tacit knowledge is needed. According to our knowledge, which is confirmed by Grant et al. (Grant et al. 2010), the only known system attempting to address challenges associated with the use of tacit knowledge is DIET system introduced by U.S. Environmental Protection Agency (Euzenat and Shvaiko 2013). DIET is built around production rules as expert system. Although the operation efficiencies of DIET are not known, the limitations of production rules when dealing with higher level of tacit knowledge or attempting to share are well known and have been proven in practice (Turban and Aronson 2001).

Following on previous development and use of ontology to model both explicit and tacit knowledge in the domain of IS and hence to support the process of IS (Trokanas et al. 2014), this paper proposes a new framework to synthesise IS networks optimised to improve environmental performance. The proposed framework is built around the ontology used to i) model tacit knowledge in the domain of IS including todate advances in resource (waste) and solution (technology) classifications, and ii) model explicit knowledge which includes expanded set of environmental and physical properties of waste and known and potential technological solutions, as well as the set of respective and commonly used environmental metrics. Tacit knowledge is built in the structure of ontology, i.e. subsumption and object properties with respective restrictions (Raafat et al. 2013). From explicit knowledge perspective, ontology is used for collecting and storing data on IS entities (participants) presented as the ontology instances. In addition, the proposed ontology enables instance matching, expanding knowledge base, generating new knowledge in the process of IS operation and knowledge sharing. Designed ontology is prepared to grow. The matching algorithm developed to match IS entities and to identify IS opportunities based on their I/O characteristics and the set of operational characteristic (Trokanas et al. 2014) is further expanded to account for environmental properties and to allow for autonomous and recursive operation towards synthesis of innovative and more complex IS networks with more than two participants. Innovative solutions are also generated by decomposition of properties characterising resources and solutions, the process optimised for environmental savings beyond inherent benefits associated with IS. This paper focuses on the theoretical concept of knowledge model and design of matching algorithm, as well as optimisation of property decomposition for given environmental conditions used in the process of synthesising IS networks. Practical 
implementation for IS which supports IS network integration and initialisation of IS operation is also proposed. The usefulness and operation of proposed formalism is demonstrated by two case studies.

\section{Theoretical Formulation of Industrial Symbiosis}

\subsection{IS Concept}

Industrial Symbiosis (IS) is a network of companies with the common interest to process waste and hence to trade material, energy and/or water to achieve economic, environmental and social gains. Unpredictability of waste availability sets IS into a category of opportunistic networks set on an ad-hoc principle which operate within confined geographical and environmental boundaries. An IS network can have a long life-time, but then it would normally go through variability in trading. The key to establishing a network is the discovery of matching participants, the process known as input/output $(1 / 0)$ matching (Trokanas et al. 2014), which fully or partially satisfy a set of technological, economic and environmental conditions and perhaps local or otherwise specified requirements. Practice suggests that full I/O matching between participants is rare and in majority of cases some adaptations are needed to satisfy partial matches (Chertow 2000). Here the term participant does not refer to an individual or a company as entity; instead it refers to the resource or solution they commit to IS. The same company can commit more than one but different resource or solution and then each of them is treated as a separate participant in IS.

1/O matching between participants, the resource (waste) providers $x_{i}$ and solution (processing technology) providers $y_{i}$, is established by matching the properties $p_{1, j}$ describing resource with the properties $p_{2, j}$ describing the input of the solution, as shown for a single matching between two participants in Figure 1. The properties $p_{i, j}$ used for matching are either descriptive, (i.e. type of material or waste composition), numerical (i.e. quantity or availability period), or even composite (i.e. pattern of availability from date to date). Still, the level of match is expected to be quantified by a single value, the similarity, which represents a measure of IS process and which is easier for comprehension by humans and for further processing merely by decision support agents.

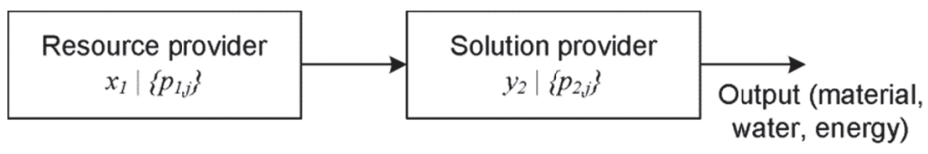

Figure 1 Principle of IS single matching

The current matching practice is manual and mediated by trained practitioners. Complex networks involving more than two partners are rare. Detailed analysis, however, shows that better cumulative IS benefits are possible with more complex IS networks where solution provider output and/or by-product become resource (input) to another solution provider (Raafat et al. 2013). One way of expanding the single matching in Figure 1 to a chained network is by recursively repeating the single matching process with each of the last solution provider in the network, $y_{2}$ in Figure 1 , switching the role to a resource provider, $x_{2}$ in Figure 2 (Trokanas et al. 2014). The whole process is repeated until all possible matches are exhausted. A cumulative measure of match, the similarity, is then calculated for comparison. Note that in this case, the match between only two participants is also considered a network. With chained matching the number of options increases exponentially with the number of participants involved.

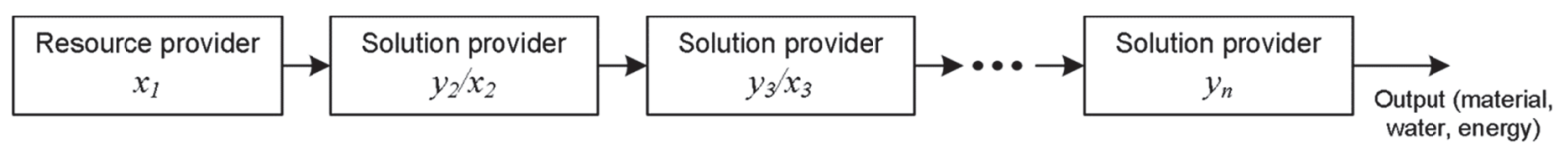

Figure 2 Principle of chained matching 
More complex networks and hence more extensive range of options are possible by introducing the decomposition of (some) properties. More precisely, some properties can be decomposed to match more than one follow-on solution provider or resource consumer. The properties referring to quantity and to period of availability of resources and solutions are perhaps the most obvious properties to decompose, as shown in Figure 3 for the first stage of matching with only one resource provider. Here, the total quantity $Q$ offered by the resource provider and characterised by the property hasQuantity is decomposed into $n$ smaller portions $q_{1}, q_{2}, \cdots, q_{n}$ such that $\sum_{n} q_{n} \leq Q$. Similarly, the whole availability period $A$ for the resource provider and characterised by the property hasAvailability is decomposed into $m$ smaller periods $a_{1}, a_{2}, \cdots, a_{m}$ which do not overlap each other and such that $\sum_{m} a_{m} \leq A$. However, with the introduction of property decomposition, the concomitant number of matching options increases multiply, as shown in Figure 4 where typically each resource provider supports more than one follow-on solution providers or resource consumer. Reversibly, it could be observed from Figure 4 that the statement that a single solution provider can be served by more than one resource providers is also valid.



Figure 3 Process of property decomposition

Several criteria for decomposing properties could be followed: i) to utilise resources characterised by decomposed property to the highest level, i.e. to maximise quantities or utilisation of availability period committed to IS; ii) to satisfy the need of most follow-on solution providers, and iii) to maximise/minimise specified cost function, i.e. to maximise aggregated economies or to maximise aggregated environmental performance of the whole network. The approach of decomposition of properties proposed in this paper is based on maximising the environmental performance. For this, we propose an ontological representation of the process of IS to orchestrate the process of property decomposition and mix-integer linear programming to optimise property decomposition for achieving the targets.

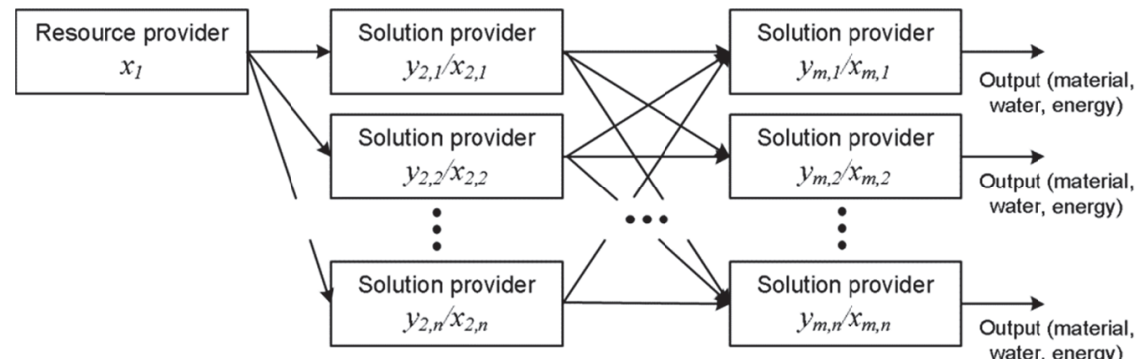




\subsection{The IS Domain Ontology}

The process of matching, hence the formation of IS networks is orchestrated by an ontology representing the IS domain (Raafat et al. 2013), e.g. resource (waste) and solution classifications, as well as the operation of IS (Trokanas et al. 2014). More precisely, the participants, namely $n_{W}$ resource providers $x=$ $\left\{x_{1}, x_{2}, \cdots, x_{n_{W}}\right\}$ and $n_{T}$ solution providers $y=\left\{y_{1}, y_{2}, \cdots, y_{n_{T}}\right\}$ are instances of the IS domain ontology, which takes format of a 6-tuple $O=\left\langle H^{I}, H_{C}, R_{i}^{C}, R^{C}, E^{C}, S_{i}^{I}\right\rangle$ consisting of:

i) $s_{i}$ instances characterised by a set of $n_{P}$ properties, $P_{i}^{n_{P}}=\left\{p_{i, j} \mid i=1, \cdots, n_{I}\right\}_{j=1}^{n_{P}}, p_{i, j} \stackrel{\text { char }}{=} s_{i}$, each representing an IS participant (resource provider or solution provider) and organised into classes $S_{i}^{I}$ as

$$
S_{i}^{I}=\left\{s_{j}\right\}_{j=1}^{n_{C}}, p_{i, j}:=p_{i, k} \wedge \forall j, k \geq 0, j \neq k
$$

where $n_{C}$ is the total number of instances sharing $n_{P}$ common properties, that is instances with intensionally equal ${ }^{1}$ properties $p_{i, j}:=p_{i, k}$. For $j=0, S_{i}^{I}$ is an empty class and still having properties $p_{i, j}$;

ii) A set of $n$ classes $H^{I}=\left\{S_{i}^{I}\right\}_{i=1}^{n}$ with each class $S_{i}^{I}$ having a distinct name $N_{i}^{I}$ hence representing a concept with respective semantic. As all instances $s_{j}$ of a class $S_{i}^{I}$ share the common properties (see eq. (1)), then the set of properties $P_{i}^{n_{P}}$ semantically describes the class $S_{i}^{I}$. Consequently, the intension $I_{i}^{I}$ of the class $S_{i}^{I}$ is defined as 3 -tuple (Junli et al. 2006);

$$
I_{i}^{I}:=\left\langle N_{i}^{I}, P_{i}^{n_{P}}, S_{i}^{I}\right\rangle
$$

iii) A graph $H_{C}=\left(S_{i}^{I}\right.$, is $\left.-a\right)$ forming a subsumption hierarchy in ontology sense, called the subsumption, were $i s-a$ indicates the edge between the nodes of the graph representing the classes (or concepts). As such, the edge $i s-a$ represents class $\left(S_{k}^{I}\right)$ - subclass $\left(S_{i}^{I}\right)$ participation which assumes property inheritance (from a subclass to a class), such that

$$
S_{i}^{I} \subseteq S_{k}^{I}, \forall P_{k}^{n_{P}} \subseteq P_{i}^{n_{P}} \wedge i \neq k
$$

In other words, instances of a subclass are also instances of the class, and also all the properties $P_{k}^{n_{P}}$ of a class $S_{k}^{I}$ are inherited by the subclass $S_{i}^{I}$. The two non-empty subclasses $S_{l}^{I}$ and $S_{m}^{I}$ are disjoint classes, if they do not share any instance, that is if $S_{l}^{I} \cap S_{m}^{I}=0, \forall l \neq m$;

iv) The class relationship $R_{i}^{C}$ which is a set of bijective relationships $r_{i, j}$ between all elements of domain class $S_{i}^{I}$ and range class $S_{j}^{I}$ other than class-subclass participation (is $-a$ relationship) and which is defined as:

$$
R_{i}^{C}=\left\{r_{i, j}\left(S_{i}^{I}, S_{j}^{I}\right) \mid \forall\left(\left(S_{i}^{I}, S_{j}^{I}\right) \in H^{I}, i \neq j\right)\right\}
$$

where the term $r_{i, j}\left(S_{i}^{I}, S_{j}^{I}\right)$ refers to a predicate calculus form and hence further enhances the semantic of the ontology and forms the base for (tacit) knowledge representation;

v) $n_{R}$-dimensional subsumption $R^{C}$ of properties $R_{i}^{C}$ defined as

$$
R^{C}=\left\{r_{i . j}\left(S_{i}^{I}, S_{j}^{I}\right) \mid \forall i \neq j\right\}_{i, j=1}^{n_{R}}
$$

Note here that although the inclusion mapping $i=j$ in eq. (4) and (5) is generally possible, we exclude such a reflexive relationship for the purpose of simplifying the process without limiting practical aspect

\footnotetext{
${ }^{1}$ Two instances are intensionally equal, if they have the same structure of the properties, not necessarily the same property values.
} 
of the application in mind. For $r_{i, j}^{-1}$ being inverse instant relationship of $r_{i, j}$, then $R_{i}^{C^{-1}}$ $\left(=\left\{r_{i, j}^{-1}\left(S_{j}^{I}, S_{i}^{I}\right) \mid \forall\left(\left(S_{j}^{I}, S_{i}^{I}\right) \in S, i \neq j\right)\right\}\right)$ is the inverse class relationship of $R_{i}^{C} ;$

vi) Extension $E^{C}$ of a class $S_{i}^{I}$ which is defined by the relationship $R_{i}^{C}$ which profiles the structural properties of the class by its relations with other classes (Junli et al. 2006). For $S_{i}^{D}$ being a subset of relationship domain $S_{i}^{D}$ and $S_{i}^{R}$ being a subset of relationship range $S_{i}^{I}$, then the restriction of $S_{i}^{I}=\operatorname{dom}\left(R_{i}^{C}\right)$ to $S_{i}^{D}$ is the partial function $f_{D}=\left.\operatorname{dom} R_{i}^{C}\right|_{S_{i}^{R}}$ providing inclusion mapping $S \stackrel{f_{D}}{\rightarrow} S$ as

$$
f_{D}: S_{i}^{I} \stackrel{f_{D}}{\rightarrow} S_{i}^{D}
$$

and the restriction of $S_{j}^{I}=\operatorname{rang}\left(R_{i}^{C}\right)$ to $S_{j}^{R}$ is the partial function $f_{R}=\left.\operatorname{rang} R_{i}^{C}\right|_{S_{i}^{D}}$ providing inclusion $\operatorname{map} S \stackrel{f_{R}}{\rightarrow} S$ as

$$
f_{R}: S_{j}^{I} \stackrel{f_{R}}{\rightarrow} S_{j}^{R}
$$

In consequence, $f_{D}$ (and $f_{R}$ ) establishes the binary relationship between:

1. Domain class $S_{i}^{D}$ and range class $S_{j}^{R}$ based on universal and existential quantifiers over properties $R_{i}^{C}$ of $S_{i}^{I}$,

2. Doman class $S_{i}^{D}$ and $n, n \in \mathbb{N}$, based on cardinality quantifiers over properties $P_{i}^{n_{P}}$ of $S_{i}^{I}$, and

3. Domain class $S_{i}^{D}$ and $v, v \in s_{i} \vee N$, based on equality quantifiers over properties $P_{i}^{n_{P}}$ of $S_{i}^{I}$.

For $R_{i}^{C}$ and $R_{j}^{C}$ being the extensions of classes $S_{i}^{I}$ and $S_{j}^{I}$, respectively, then $S_{i}^{I}$ and $S_{j}^{I}$ are equivalent classes, if $R_{i}^{C}=R_{j}^{C}$ and if $S_{i}^{I} \cap S_{j}^{I}=S_{i}^{I} \cup S_{j}^{I}$.

\subsection{Matching}

Matching between participants, which are in the ontology represented by respective instances, is performed on the request of one of them, the requester, and based on i) metrics defined over properties $P_{i}^{n_{P}}=\left\{p_{i, j} \mid i=1, \cdots, n_{I}\right\}_{j=1}^{n_{P}}$ characterising them (only numerical properties will be considered for this type of matching) and representing explicit IS knowledge, and ii) metric defined over mutual position (distance) of respective classes in the ontology and representing tacit IS knowledge. In general, for a h-metric $h_{i}$ defined over set of (only) $N$ numerical properties $P_{i}^{N}=\left\{p_{i, j} \mid p_{i, j} \in \mathbb{N}, i=1, \cdots, n_{I}\right\}_{j=1}^{N} \subseteq P_{i}^{n_{p}}$ as

$$
h_{i}: P_{i}^{N} \stackrel{h}{\rightarrow} \mathbb{R}
$$

the object $\left(P_{i}^{N}, h_{i}\right)$ forms a metric space. By observing the set of numerical properties $P_{i}^{N}$ as an $N$ dimensional vector $\mathbf{p}_{i}=\left(p_{i, 1}, p_{i, 2}, \cdots, p_{i, N}\right)$, objects $\left(P_{i}^{N}, \mathbf{p}_{i}\right)$ form the vector space $Q^{N}$ of $n_{I}$ vectors. To this end, every pair of classes $\left(S_{i}^{I}, S_{j}^{I}\right)$ and/or pair of vectors $\left(\mathbf{p}_{i}, \mathbf{p}_{j}\right)$ can be mapped as $S^{2} \stackrel{h}{\rightarrow} \mathbb{R}$ :

$$
h: S \times S \stackrel{h}{\rightarrow} h\left(s_{i}, s_{j}\right) \equiv h_{i, j} \in \mathbb{R}
$$

For matching the properties, we define a h-metric $h_{k}^{V}$ over the vector space $Q^{n_{I}}$ as mapping from $\mathbb{R}^{n_{I}} \rightarrow \mathbb{R}$ so that

$$
h_{k}^{V}: Q^{n_{I}} \stackrel{h}{\rightarrow} h\left(\left(\mathbf{p}_{i}, \mathbf{p}_{j}\right)\right)
$$

\footnotetext{
${ }^{2}$ In linear algebra, a vector space is a set $V$ of vectors together with the operations of addition and scalar multiplication (and also with some natural constraints such as closure, associativity, and so on).
} 
Then, the similarity measure of the object $\left(Q^{n_{I}},\left(\mathbf{p}_{i}, \mathbf{p}_{j}\right)\right)$ is

$$
h_{k}^{V}=\left\{\frac{\mathbf{p}_{i} \cdot \mathbf{p}_{j}}{\left\|\mathbf{p}_{i}\right\|\left\|\mathbf{p}_{j}\right\|}\right\}_{i, j=1}^{n_{I}}, k=1,2, \cdots, 2^{n_{I}}
$$

For matching the position of respective classes $S_{i}^{I}$ and $S_{j}^{I}$ in the ontology, we define a h-metric $h_{k}^{C}$ over the set of classes $H^{I}$ as mapping from $S^{n_{I}} \rightarrow \mathbb{R}$ so that

$$
h_{k}^{C}: H^{I} \stackrel{h}{\rightarrow} h\left(\left(S_{i}^{I}, S_{j}^{I}\right)\right)
$$

Then, the similarity measure of the object $\left(H^{I},\left(S_{i}^{I}, S_{j}^{I}\right)\right)$ is

$$
h_{k}^{C}=\min _{S_{C}^{I} \in H^{I}}\left[\delta\left(S_{i}^{I}, S_{C}^{I}\right)+\delta\left(S_{j}^{I}, S_{C}^{I}\right)\right]
$$

where $\delta\left(S_{i}^{I}, S_{C}^{I}\right)\left(\delta\left(S_{j}^{I}, S_{C}^{I}\right)\right)$ is the distance between classes $S_{i}^{I}\left(S_{j}^{I}\right)$ and another class $S_{C}^{I}$ within the set $H^{I}$ measured in number of intermediate edges ${ }^{3}$ in graph sense along subsumption $H_{C}$ and along selected $R_{i}^{C}$ relationships. The process of selecting which relationship $R_{i}^{C}$ to use is application dependent (not all relationships $R_{i}^{C}$ are necessarily used) and in this work we use properties defined in Table 4.

For an unambiguous quantification of a match between two instances representing IS participants we aggregate similarity measures $h_{k}^{V}$ and $h_{k}^{C}$ as

$$
h_{k}=\frac{\alpha h_{k}^{V}+\beta h_{k}^{C}}{\alpha+\beta}
$$

where $\alpha$ and $\beta$ are weighting factors deepening the semantics of the ontology similarity and their values are dictated by the application. More specifically, equation (14) indicates aggregation of tacit similarity expressed by eq. (13) and explicit similarity expressed by eq. (11) into a single similarity measure characterising the match between two instances.

\subsection{Modelling of Environmental Effects}

In the IS domain ontology, three major groups of properties are used to characterise both resources (waste) and solutions (technologies) and hence to enable assessment of economic $\left(n_{E c}\right.$ properties $\left.P_{i}^{n_{E c}}=\left\{p_{i, j} \mid p_{i, j} \in \mathbb{N}, i=1, \cdots, n_{I}\right\}_{j=1}^{N_{E c}} \subseteq P_{i}^{n_{p}}\right), \quad$ environmental $\quad\left(n_{E n} \quad\right.$ properties $\left.P_{i}^{n_{E n}}=\left\{p_{i, j} \mid p_{i, j} \in \mathbb{N}, i=1, \cdots, n_{I}\right\}_{j=1}^{n_{E n}} \subseteq P_{i}^{n_{p}}\right) \quad$ and $\quad$ operational $\quad\left(n_{O p} \quad\right.$ properties $\left.P_{i}^{n_{O p}}=\left\{p_{i, j} \mid p_{i, j} \in \mathbb{N}, i=1, \cdots, n_{I}\right\}_{j=1}^{n_{O p}} \subseteq P_{i}^{n_{p}}\right)$ benefits, as shown in Table 1. In Table 1 the semantic of the properties is self-explanatory by their names (a full description is provided in Appendix $C$ ) and the superscript ${ }^{(\mathrm{N})}$ indicates numerical properties.

Table 1 Properties $p_{i, j}$ used in the IS domain ontology

\begin{tabular}{lcccccc}
\hline \multirow{2}{*}{ Property name } & \multirow{2}{*}{$\begin{array}{c}\text { Operational } \\
\text { features }\end{array}$} & \multirow{2}{*}{$\begin{array}{c}\text { Environmental } \\
\text { features }\end{array}$} & $\begin{array}{c}\text { Economic } \\
\text { features }\end{array}$ & & Resources & Solutions $\begin{array}{c}\text { IS } \\
\text { operation }\end{array}$ \\
\hline hasQuantity $^{(N)}$ & $v$ & $v$ & & $v$ & $v$ \\
hasProcessingPrice $^{(N)}$ & & $v$ & $v$ & $v$ & $v$ \\
hasAnnualCost $^{(N)}$ & & $v$ & $v$ & $v$ & \\
isValidFrom $^{(N)}$ & $v$ & & & $v$ & $v$ \\
\end{tabular}

\footnotetext{
${ }^{3}$ The term edge represents the links or relationships between the two classes in the graph $H_{C}$.
} 


\begin{tabular}{|c|c|c|c|c|c|c|}
\hline isValidTo $^{(N)}$ & $v$ & & & $v$ & $v$ & \\
\hline hasName & $v$ & & & $v$ & $v$ & \\
\hline isBiodegradable & $v$ & $v$ & & $v$ & & \\
\hline isHazardous & $\checkmark$ & $v$ & & $v$ & & $v$ \\
\hline embodiedCarbon $^{(N)}$ & $v$ & & & $v$ & & \\
\hline hasCO2emission $^{(N)}$ & & $v$ & $v$ & & $v$ & \\
\hline hasByProduct & $v$ & $v$ & & & $v$ & \\
\hline needsEnergy ${ }^{(N)}$ & $v$ & $v$ & & & $v$ & \\
\hline needsWater ${ }^{(N)}$ & $v$ & $v$ & & & $v$ & \\
\hline hasConversionRate $e^{(N)}$ & $v$ & $v$ & & & $v$ & \\
\hline$g e o: L a t^{(N)}$ & $v$ & & $v$ & & & $v$ \\
\hline geo:Long ${ }^{(N)}$ & $v$ & & $v$ & & & $v$ \\
\hline belongTolndustry & & $v$ & $v$ & & & $v$ \\
\hline hasStorageCapacity $^{(N)}$ & $v$ & & & & & $v$ \\
\hline hasStorageMethod & $v$ & & & & & $v$ \\
\hline hasDeliveryMethod & $v$ & & $v$ & & & $v$ \\
\hline
\end{tabular}

The environmental performance of IS networks is evaluated by five metrics calculated from environmental properties in Table 1 and they include: i) landfill diversion savings $L D S$, ii) embodied carbon impact $E C I$, iii) transportation impact TI, and iv) virgin materials saved VMS. Calculation of all five metrics is in detail explained in reference (Trokanas et al. 2015) and summarised in Table 2.

Table 2 Calculation of environmental metrics

\begin{tabular}{|c|c|c|}
\hline Metric & Calculation & Variables \\
\hline $\begin{array}{l}\text { Landfill } \\
\text { diversion }\end{array}$ & $L D S=\sum^{\text {Resource }} Q_{i, j} *\left(D C_{i, j}+L T\right)$ & $\begin{aligned} Q_{i, j}- & \text { quantity of exchanged resources, the } \\
& \text { ontology property hasQuantity, }\end{aligned}$ \\
\hline savings $L D S$ & $\sum_{i, j}$ & $D C_{i, j}$ - the disposal cost of the resource (waste), \\
\hline $\begin{array}{l}\text { Embodied } \\
\text { carbon impact } \\
\text { ECI }\end{array}$ & $E C I=\sum_{i, j}^{\text {Resource }}\left(Q_{i, j} * E C_{i, j} * C O_{2}^{P}\right)$ & $\begin{array}{l}L T \text { - the landfill tax, } \\
E C_{i, j} \text { - resource embodied carbon value, the } \\
\quad \text { ontology property embodiedCarbon, } \\
C O_{2}^{P} \text { - the carbon dioxide credit price, }\end{array}$ \\
\hline $\begin{array}{l}\text { Transportation } \\
\text { impact } T I\end{array}$ & $T I=\sum_{i, j}^{\text {synergy }}\left(Q_{i, j} * T F_{i, j} * D_{i, j}^{R} * C O_{2}^{P}\right)$ & $\begin{array}{l}T F_{i, j} \text { - the factor characterising the emission of } \\
\text { particular mode of transportation, the } \\
\text { ontology property hasDeliveryMethod, } \\
D_{i, j}^{R}-\text { the distance between IS participants } i \text { and } j\end{array}$ \\
\hline \multirow[t]{3}{*}{$\begin{array}{l}\text { Virgin } \\
\text { materials } \\
\text { saved VMS }\end{array}$} & $V M S=\sum_{i, j}^{\text {synergy }} C_{i, j} *\left(F P_{i, j}-R P_{i, j}\right)$ & $\begin{array}{l}\text { calculated from geographical longitude and } \\
\text { latitude of participants (the ontology } \\
\text { properties geo:Lat and geo:Long), }\end{array}$ \\
\hline & & $\begin{array}{l}C_{i, j}-\text { committed resource capacities between the } \\
\text { participants } i \text { and } j,\end{array}$ \\
\hline & & $\begin{array}{l}F P_{i, j} \text { - price of the resource as a raw material, } \\
R P_{i, j} \text { - price of the resource as a recyclate. }\end{array}$ \\
\hline
\end{tabular}

\subsection{Optimisation of Property Decomposition}

In the process of property decomposition we have adopted the following principles which assure generation of technologically logical and operationally viable IS network options:

i) Only numerical properties are used for decomposition. In this paper we propose decomposition of resource quantity (the ontology property hasQuantity) and resource availability (the ontology properties isValidFrom and isValidTo). The property decomposition only involves matches which have semantic 
similarity $h_{k}$ higher than the threshold value $h_{k}^{\text {tres }}$ defined by the matching requestor (default value is $\left.h_{k}^{\text {tres }}=0.5\right)$. Matches with $h_{k}<h_{k}^{\text {tres }}$ are eliminated as options altogether;

ii) Each portion of the split part of resources quantity (the ontology property hasQuantity) cannot be smaller than the economical level $r_{1, f}$ defined by the match requester. The minimum quantity normally depends on the economies dictated by the transportation and/or switching cost of processing in case where the resources from different participants are not of exactly the same type hence includes transition from one resource to another;

iii) The time of availability is only decomposed to the level of satisfying the full overlap between the participants and to fill-in remaining availability gap, as shown in Figure 5. Further decomposition of established availability overlap is not considered, e.g. Partner 3 in Figure 5 is not considered because its inclusion would violate the principle of 'full overlap' only;

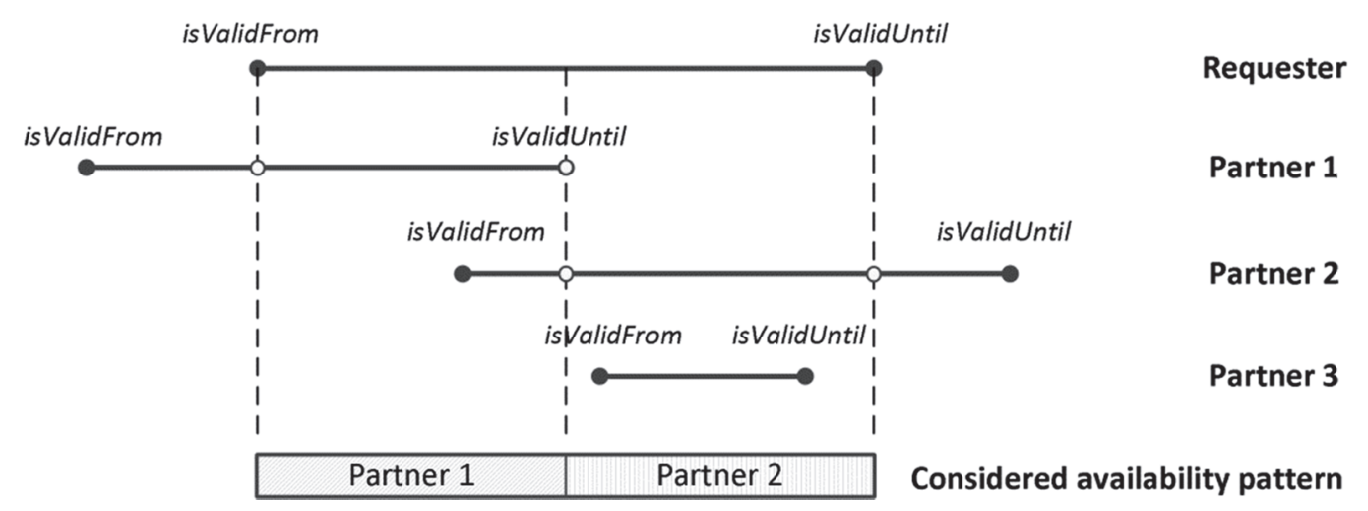

Figure 5 Decomposition constrain of the availability period

iv) Each availability time portion cannot be shorter than the time fence ET defined by the requester and which is normally dictated by the transportation requirements and/or switching process and respective costs. It has to be noted here that the transportation requirements are case specific and not modelled in the ontology for the purpose of optimisation. Similarly, the switching between individual time sequences involving different resources are resource-technology dependent, by enlarge unknown in practice and it is a topic of current research;

v) The matching requester could be either 1 ) resource provider when a forward matching process is initiated which results in a single resource used to provide different end-products (Figure 6a), or 2) solution provider when a backward matching process is initiated with more than one resources combined to result in a single end product (Figure $6 \mathrm{~b}$ ). Combination of the two matching requesters is not considered;

vi) There is a single network solution, the prime solution, which includes all the partners satisfying the technological and IS logic and hence included in principles i) to iv). All other solutions are sub-networks of the prime solution, as demonstrated in Figure 6. 




Figure 6 Process of property decomposition to support IS network formation

Following the property decomposition principles i) to vi), a partial matching is used to form the prime IS solution, namely the prime network, first. More precisely, partial matching is possible by using only part(s) of properties hasQuantity for the resources quantity and solution capacities and hasAvailability for the resource/solution availability period determined from isValidFrom and isValidTo properties (Table 1) in this case, with the property decomposition defined as:

$$
\sum_{k=1}^{K} r_{i, k}\left(S_{i}^{I}, k\right) \leq r_{i}^{I}
$$

where $K$ denotes the decomposition level of the property $r_{i, k}$ referring to the participants $i$. The optimal level of decomposition for each matching option between two participants in the network is achieved by applying the mixed integer linear programming (MILP) optimisation of the general form

$$
\begin{array}{cc}
\text { maximise } & E N V I=f\left(r_{i, 1}, r_{i, 2}\right) \\
\text { s.t. } & h\left(r_{i, 1}, r_{i, 2}\right)=0 \\
& g\left(r_{i, 1}, r_{i, 2}\right) \leq 0 \\
& y \in\{0,1\}
\end{array}
$$

where the objective function $f$, as well as constraints $h$ and $g$, are all functions of properties $r_{i, 1}$ and $r_{i, 2}$ which refer to properties hasQuantity and hasAvailability, respectively, and the set of variables $y$ defines existence of properties and/or portion of properties in relation to the decomposition principles i) to vi). Here, the objective function ENVI refers to environmental effects defined by environmental metrics in Table 2 as

$$
E N V I=L D S-E C I-T I+V M S
$$

The constraint functions $h, g$ in eq. (16) account for the problem constrains assuring that decomposition principles i) to vi) are satisfied in addition to general principle of IS operation and respective technological constraints as shown in Table 3. As such, the constraints $h$ and $g$ define the feasibility area consistent with the resources, namely quantities $r_{i, 1}$ and availability $r_{i, 2}$ (Table 3). Along with the quantity $r_{1, i}$ consistency, the switching time constraint is used to eliminate any participation which does not cover the time fence ET set by the requester. The default value of the time fence $E T_{R e q}$, if not provided by the requester, is calculated as a percentage of the total availability period with $20 \%$ used as a guideline from practice: 


$$
E T_{\text {Req }}=\left(\text { isValidTo }_{\text {Request }}-\text { isValidFrom }_{\text {Request }}\right) * 0.2
$$

And finally, the quantity decomposition is also governed by the environmentally sound quantity $E Q$ (Table 3). For the time horizon $T_{H}$ (default value is set to 1 month), the environmentally sound quantity is calculated as

$$
E Q_{i}=\frac{r_{1, i}}{A_{i}}
$$

where $r_{1, i}$ is committed quantity and $A_{i}$ is availability period calculated as

$$
A_{i}=\left(\text { isValidTo }_{i}-\text { isValidFrom }_{i}\right)
$$

The environmentally sound quantity of the request has to be satisfied by the sum of the available quantities;

$$
\mathrm{EQ}_{\text {Request }} \leq \sum \mathrm{EQ}_{\mathrm{i}}
$$

\begin{tabular}{|c|c|c|}
\hline Metric & Calculation & Variables \\
\hline $\begin{array}{l}\text { Request quantity } \\
\text { constraints }\end{array}$ & $\sum_{i}^{n_{i i}} r_{1, i} \leq r_{1, r}$ & $\begin{array}{ll}r_{1, i}- & \text { available quantities of participating resource } \\
& \text { providers, } \\
n_{i j}- & \text { number of participating resource providers }\end{array}$ \\
\hline $\begin{array}{l}\text { Resource provider } \\
\text { quantity } \\
\text { constraints }\end{array}$ & $r_{1, i} \leq r_{1, i}^{c}, \forall i$ & $\begin{array}{ll}r_{1, r}- & \text { requested quantity } \\
r_{1, c}- & \text { committed quantities by resource provider } i,\end{array}$ \\
\hline $\begin{array}{l}\text { Economic level } \\
\text { quantity } \\
\text { constraints }\end{array}$ & $r_{1, i} \geq r_{1, f}, \forall i$ & $\begin{array}{l}\text { defined by requester (see decomposition } \\
\text { principle ii) }\end{array}$ \\
\hline $\begin{array}{l}\text { Switching time } \\
\text { constraint }\end{array}$ & $E T_{\text {Req }} \geq$ Overlap $_{\text {Request }, i}$ & \multirow{2}{*}{$\begin{array}{l}E T_{R e q} \text { - acceptable switching time for Requester } \\
\text { Overlap } \\
\text { usequest }, i \text { - overlap between request and } \\
E Q_{i} \text { - economic quantity for user } i \text {. }\end{array}$} \\
\hline $\begin{array}{l}\text { Economic quantity } \\
\text { constraint }\end{array}$ & $E Q_{\text {Request }} \leq \sum E Q_{i}$ & \\
\hline
\end{tabular}

Table 3 Specification of the optimisation constraints

In order to provide intuitive results understood by users and comparable to the similarity measure (14), optimised environmental indicator ENVI from eq. (17) is normalised to the range defined by maximum $E N V I_{\max }$ and minimum $E N V I_{\min }$ values as

$$
E N V I_{i, j}^{\text {normalised }}=\frac{E N V I_{i, j}-E N V I_{\min }}{E N V I_{\max }-E N V I_{\min }}
$$

which aggregated with the similarity $h_{k}$ (eq. (14)) gives the network suitability measure $M_{n w}$ as

$$
M_{n w}=07 \cdot h_{k}+0.3 \cdot E N V I_{i, j}^{\text {normalised }}
$$

\subsection{Description of the Algorithm for Optimisation of Environmental Properties}

In its full implementation, the process of synthesising environmentally optimised IS networks starts with identifying all technologically viable options satisfying the request, the process fully controlled by the IS domain ontology and respective semantic matching. The whole process is outlined in Figure 7 and takes the following form:

Step 1: Execute semantic matching recursively at each level of the network, starting from the requester and using i) forward matching for resource (waste provider) as requestor (Figure 6a), or, ii) backward matching for solution (technology provider) as requestor (Figure 6b). Identify all subnetworks satisfying technological relevance and rank them by semantic relevance using aggregated similarity calculated by eq. (14) and for all matching partners in the network; 
Step 2: From all options identified in step 1 select the prime network, the network containing all other networks. Remove all the individual participant pairs with semantic similarity $h_{k}$ below set threshold $h_{k}^{\text {tres }}$, that is $h_{k}<h_{k}^{\text {tres }}$, and identify all $n_{n}$ remaining network participants and their solution providers in case of forward matching or resource providers in case of backward matching. Note that in case of forward matching $n_{n t}$ terminal participants do not have solution providers, as well as in case of backward matching $n_{n i}$ initial participants do not have resource providers;

Step 3a: For forward matching, calculate optimised decomposed quantities $r_{i, 1}$ (hasQuantity) and time availability $r_{i, 2}$ (has Availability) for all $n_{n}-n_{n t}$ non-terminal network participants $x_{k}$ and for all $i$ of their matching solution providers $y_{i}$, as defined by equations (17), (18) and (19) and by assuring that condition (21) is satisfied;

Step 4b: For backward matching, calculate optimised decomposed quantities $r_{i, 1}$ (hasQuantity) and time availability $r_{i, 2}$ (has Availability) for all $n_{n}-n_{n i}$ non-initial network participants $y_{k}$ and for all $i$ of their matching resource providers $x_{i}$, as defined by equations (17), (18) and (19) and by assuring that condition (21) is satisfied;

Step 5: Calculate aggregated network similarity for decomposed properties $r_{i, 1}$ and $r_{i, 2}$ and compare with the semantic similarity $h_{k}$ of every other network proposed in step 1.

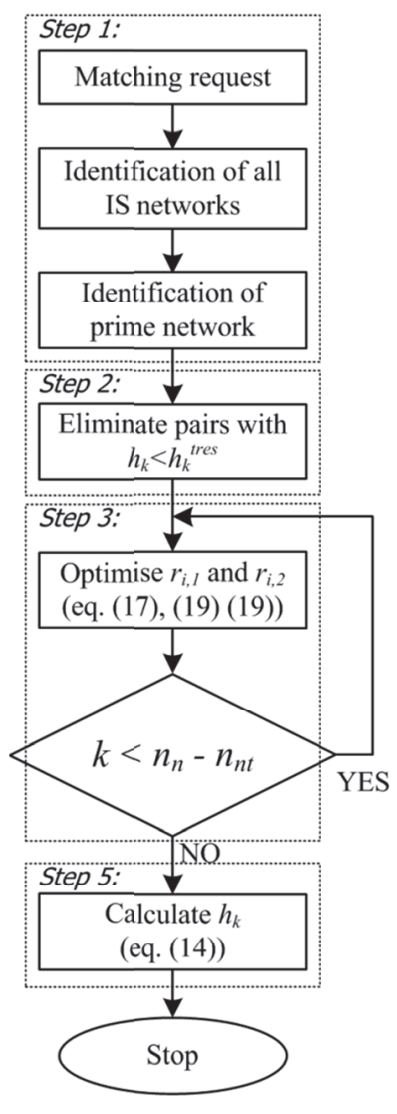

a) forward matching

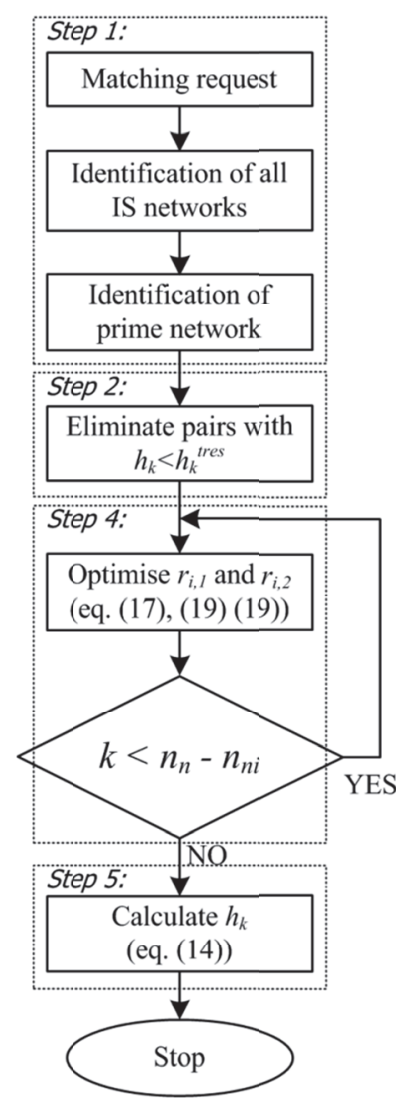

b) backward matching

Figure 7 Optimisation of parameter decomposition algorithm flow diagram

\section{Implementation}

The proposed formalism and respective decomposition algorithm explained in Section 2.6 are implemented as a web service which includes IS domain ontology and I/O matching engine, both functionality integrated 
by a web portal (Cecelja, F. et al. 2014b) and accessible through a user friendly interface, as demonstrated in Figure 8.

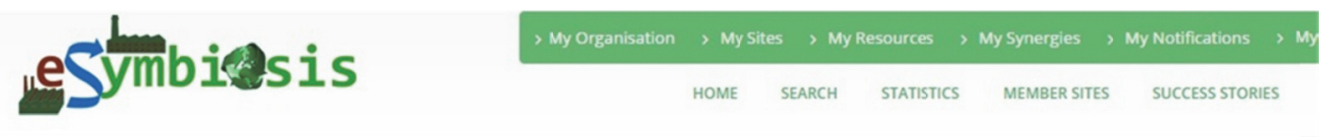

Register a Resource

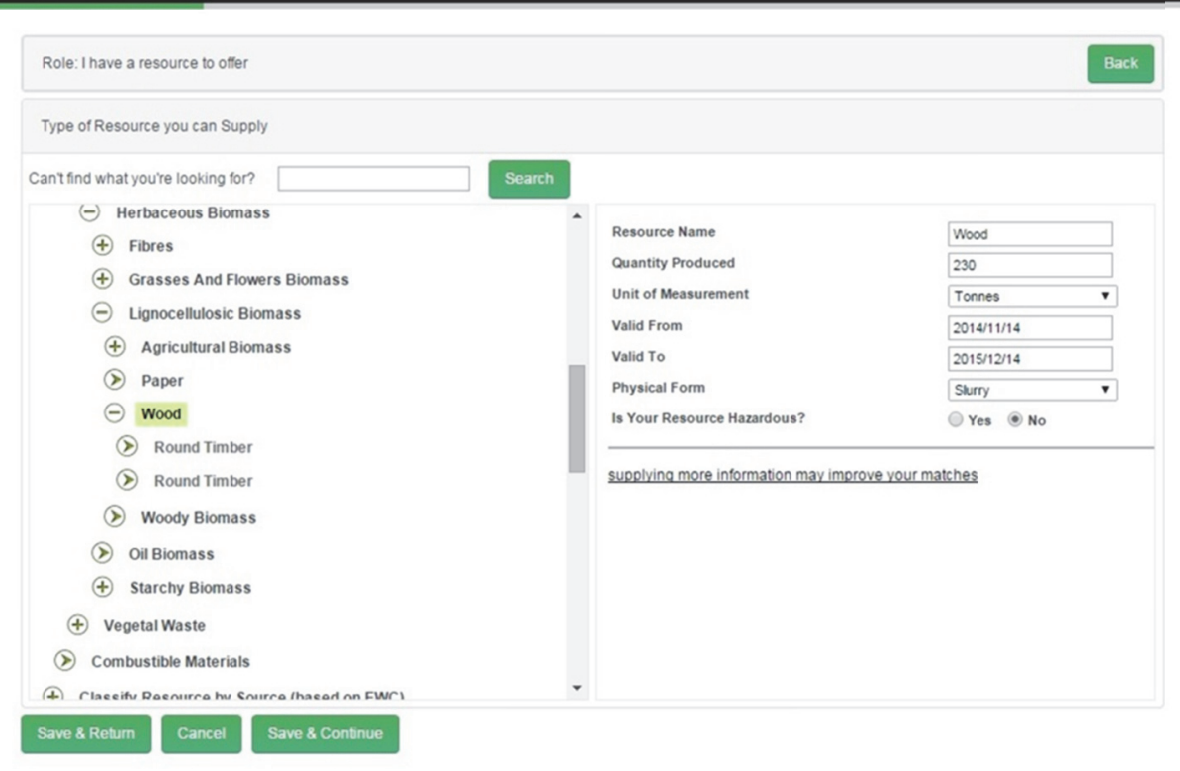

Figure $8 \mathrm{~A}$ user friendly interface of the web service implementation

\subsection{IS Domain Ontology}

Used for knowledge representation, ontologies, as defined in Section 2.2, are built upon classes $S_{i}^{I}$ with unique names $N_{i}^{I}$ organised in subsumption $H_{C}$ which organise instances $s_{i, j}$ with common properties $p_{i, j}$ and which have relationships $R_{i}^{C}$ between them. Here, instances are IS participants including the resources, the waste providers, and solutions, the technology providers, as defined by eq. (1). For the ultimate function of matching inputs and outputs in IS sense and with the inclusion of partial matching, and hence forming symbiotic networks, the ontology enables;

1. Modelling of tacit IS knowledge by the selection of unique concept names $N_{i}^{I}$, structure of subsumtion $H_{C}$ and by selection of relationships $R_{i}^{C}$ and respective restrictions. In consequence, the ontology supports identification of potential synergies by providing a common reference;

2. Modelling of explicit IS knowledge through dynamic instantiation and increasing the population, hence enabling the ontology to grow, and;

3. Supporting the process of $\mathrm{I} / \mathrm{O}$ matching.

The instantiation and increasing the population in the set of instances $S$ is the key function of the ontology. For that, the ontology models the knowledge in the domain of IS enabling both resources and solutions to be instantiated with values of the properties $p_{i, j}$ collected through the process of user registration. The whole registration process is navigated by parsing ${ }^{4}$ the inferred ${ }^{5}$ ontology.

\footnotetext{
${ }^{4}$ Parsing the ontology is the process of analysing it to determine its structure with respect to subsumption and other relationships between classes and hence to determine interdependence or to extract properties.

${ }^{5}$ The process of inference refers to the process of inferring the restrictions on properties, as defined in Section 2.1.
} 
Common referencing is established by formalising tacit knowledge of IS in the form of ontology structure. The common vocabulary, representing the semantic by selection of concept names $N_{i}^{I}$, is identified to include resources, i.e. materials, products, technologies, and peripheral information needed for the solutions, i.e. regions, units of measurement and measure qualifications, among others. The identification of the IS terminology establishes a standardized vocabulary for IS and eliminates syntactic differences in the process of I/O matching. In the current implementation (Raafat et al. 2013), we use vocabulary established by European Commission in the form of the European Waste Catalogue (EWC) (EC 2002), and others that can enhance the process such as material type, processing technologies and other waste stream classifications including EWC STAT (EC 2010).

The developed ontology has four levels of abstraction (Trokanas et al. 2014) covering the domain of IS: i) meta-level which defines general purpose concepts of the ontology that can be applied universally and have no dependence on the specific domain, ii) top level providing abstract concepts of the domain as well as the top-level relationships between these concepts, iii) domain level which details the domain of IS, and iv) instantiation level which is application specific level with the user profile instances, as illustrated in Figure 9.

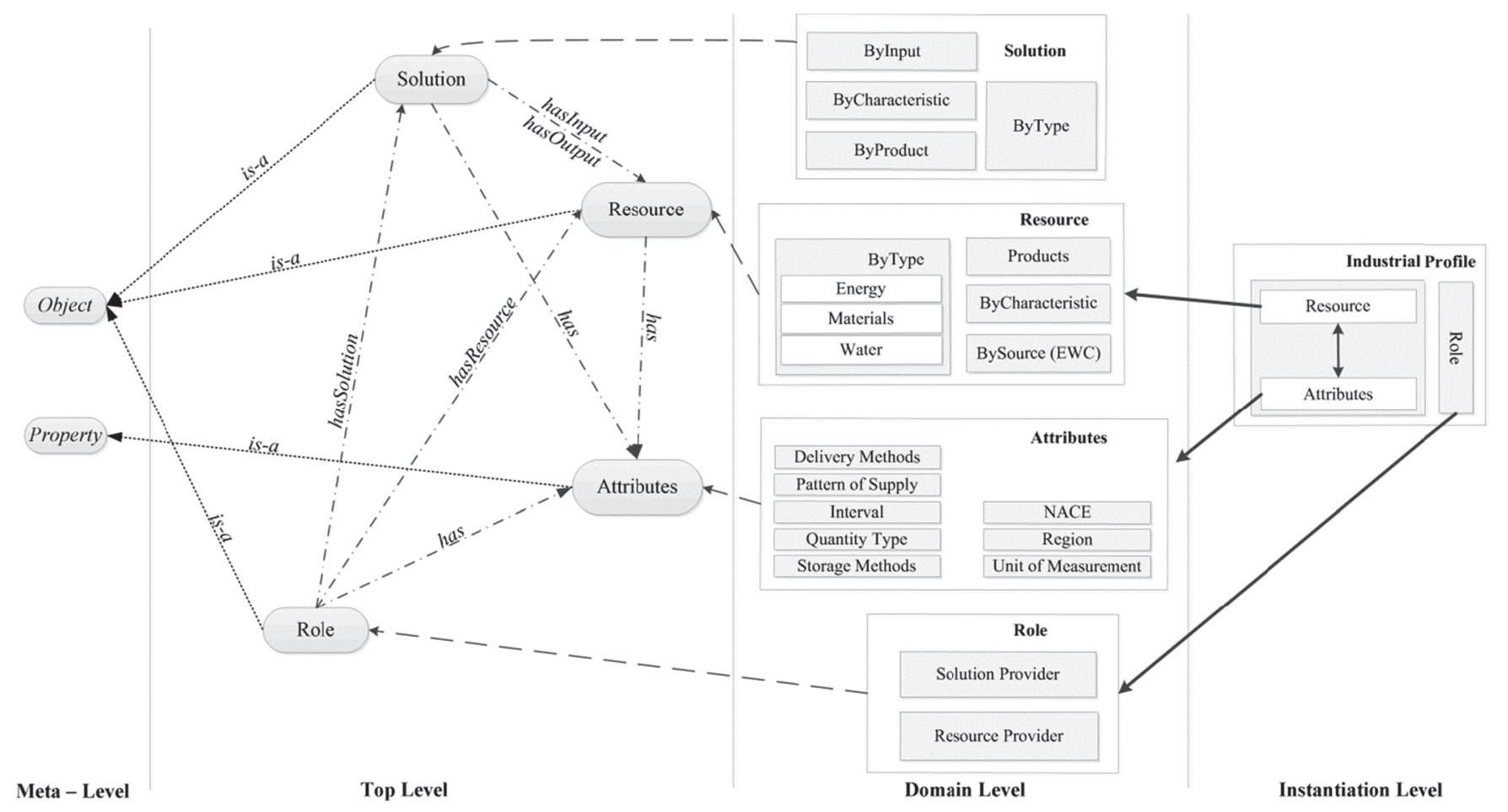

Figure 9 Ontology design

The domain level of ontology further details concepts at the top level split into the ontology modules. The classes $H^{I}$ which form modules defined at the top level are organised in the subsumption $H_{C}$ with the first four levels detailed in Appendix A. The indentation in the leftmost column of the Appendix A indicates hierarchical class - subclass $(i s-a)$ participation. The complete ontology at present contains approximately 2000 classes (Cecelja, F et al. 2014a).

Along with the properties $P_{i}^{N}$ used to characterise classes (Table 1), the set of implemented relations $R_{i}^{C}$ is given in Table 4. They are introduced to facilitate and to enhance instantiation process (participant registration), to model knowledge and to enable calculation of semantic similarity $h_{k}$ (eq. (14)). The indentation in the column Relation $\mathrm{R}_{\mathrm{i}}^{\mathrm{C}}$ in Table 4 indicates property - subproperty subsumption relationship introduced to further granulate the knowledge representation. Table 4 also illustrates domain $S_{i}^{D}$ and 
range $S_{j}^{R}$ classes for each relationship with the name of the relationships being self-explanatory and which are fully described in Appendix B.

Table 4 Set of used relations $R_{i}^{C}$

\begin{tabular}{|c|c|c|c|c|}
\hline Relation $R_{i}^{C}$ & Domain class $S_{i}^{D}$ & Range class $S_{j}^{R}$ & Use & Feature \\
\hline geo:location & Role & Geo:SpatialThing & SM & Social, Operational \\
\hline belongsTolndustry & Role & NACE & IP & Operational \\
\hline hasResource & ResourceProvider & Resource & Both & Operational \\
\hline hasTechnology & SolutionProvider & Technology & Both & Operational \\
\hline hasPatternOfSupply & Resource & PatternOfSupply & Both & Operational \\
\hline hasApplicationIn & Resource & NACE & IP & Operational \\
\hline canUse & NACE & Resource & IP & Operational \\
\hline hasQuantityType & Resource & Quantity Type & IP & Operational \\
\hline hasUnitOfMeasurement & Resource & UnitOfMeasurement & IP & Operational \\
\hline hasComposite & $\begin{array}{c}\text { Products, } \\
\text { ResourceBySource }\end{array}$ & ResourceByType & Both & Operational \\
\hline isCompositeOf & ResourceByType & $\begin{array}{c}\text { Products, } \\
\text { ResourceBySource }\end{array}$ & SM & Operational \\
\hline $\begin{array}{l}\text { hasInput } \\
\text { canProcess }\end{array}$ & Technology & Resource & Both & Operational \\
\hline canBeProcessedBy & Resource & Technology & Both & Operational \\
\hline $\begin{array}{l}\text { hasOutput } \\
\text { hasProduct }\end{array}$ & Technology & Resource & SM & Operational \\
\hline needsEnergy & Technology & Energy & IP & Environmental \\
\hline needsWater & Technology & Water & Both & Environmental \\
\hline hasStorageMethod & Resource & StorageMethods & IP & Operational \\
\hline hasDeliveryMethod & Resource & DeliveryMethods & IP & Operational \\
\hline
\end{tabular}

With reference to IS, the restrictions have a three-fold purpose; i) to provide new links between classes, ii) to enhance the flexibility of the instantiation process, and iii) to provide links to the common reference block of the ontology - the materials classification. They are all inferred by the inference engine. The restrictions used in this paper are all listed in Table 5. From Table 5, for example, the hascomposite relationship can only link the members of Products and ResourceBySource classes to the class Material. The cardinality restrictions with value range $=1$ define that these are mandatory fields for the user to fill in during the registration. Regarding the class BiodegradableResource, we define that if any resource has isBiodegradable property set to true, it will then be inferred to be a BiodegradableResource and will be reclassified under this class by inferring the ontology.

Table 5 Used restrictions

\begin{tabular}{l|c|c|c}
\multicolumn{1}{c|}{ Domain Class $S_{i}^{I}$} & Property $\operatorname{dom}\left(R_{i}^{C}\right)$ & Type & Value Range \\
\hline Role & confidentialityFlag & Cardinality & $=1$ \\
Role & geo:location & Cardinality & $=1$ \\
Role & belongsTolndustry & Range & NACE \\
ResourceProducer & hasResource & Range \& Cardinality & Resource(>1) \\
SolutionProvider & hasSolution & Range \& Cardinality & Technology (>1) \\
Resource & hasQuantity & Cardinality & $=1$ \\
Resource & hasPatternOfSupply & Cardinality & $=1$ \\
Resource & validFrom & Cardinality & $=1$ \\
Resource & validTo & Cardinality & $=1$ \\
Resource & isBiodegradable & Cardinality & $<1$ \\
BiodegradableReso & isBiodegradable & Value & true
\end{tabular}




\begin{tabular}{|c|c|c|c|}
\hline urce & & & \\
\hline $\begin{array}{l}\text { Products, } \\
\text { ResourceBySource }\end{array}$ & hasComposite & Range & Materials \\
\hline Materials & isCompositeOf & Range & $\begin{array}{c}\text { Products, } \\
\text { ResourceBySource }\end{array}$ \\
\hline Technology & canProcess & Range \& Cardinality & Resource (>1) \\
\hline Technology & hasProduct & Range \& Cardinality & Resource $(>1)$ \\
\hline Technology & needsEnergy & Range \& Cardinality & $\begin{array}{l}\text { Energy or Energy } \\
\text { Products }\end{array}$ \\
\hline Technology & needsWater & Range \& Cardinality & Water $(<1)$ \\
\hline
\end{tabular}

\subsection{I/O Matching}

The semantic matching is the process of establishing the semantic relevance between instances or parts of the ontology (Trokanas et al. 2014). The I/O matching refers to matching instances in IS ontology. The output of the matching is quantified by the similarity measure $h_{k}$, as defined by eq. (14). In practical terms, explicit knowledge is represented by values of instance properties $P_{i}^{n_{P}}$, whereas tacit knowledge is represented by the ontology structure and hence by position of instantiated class in post-inferred ontology in respect to the subsumption $H_{C}$ and relationships $R_{i}^{C}$ (and relationship hierarchy $R^{C}$ ). As such, matching of tacit knowledge enables partial establishment of technological relevance, i.e. for a technology capable of processing biodegradable material it can be inferred that it can also process straw as it is biodegradable material. Likewise, matching explicit knowledge enables quantification of matched relevance with respect to operational side of IS. In consequence, the process of I/O matching is not only bound to identify direct matches. It can semantically interpret the relationships that different concepts have with each other and it is therefore capable of suggesting alternative resource, identify technologies that process similar types of resource or propose different application for a resource. Partial matching which is seen as an important feature of IS network formation is further amplified by the property decomposition, as explained in Section 2.5 .

In the current implementation, the process of $1 / 0$ matching is performed in two stages (Figure 10): i) the stage of elimination, ii) the stage of calculating the similarity measures $h_{k}^{V}$ and $h_{k}^{C}$ between request instance and remaining (after elimination) instances, and iii) the stage of aggregating similarity measures $h_{k}^{V}$ and $h_{k}^{C}$ into a single similarity measure characterising the match between two instances.

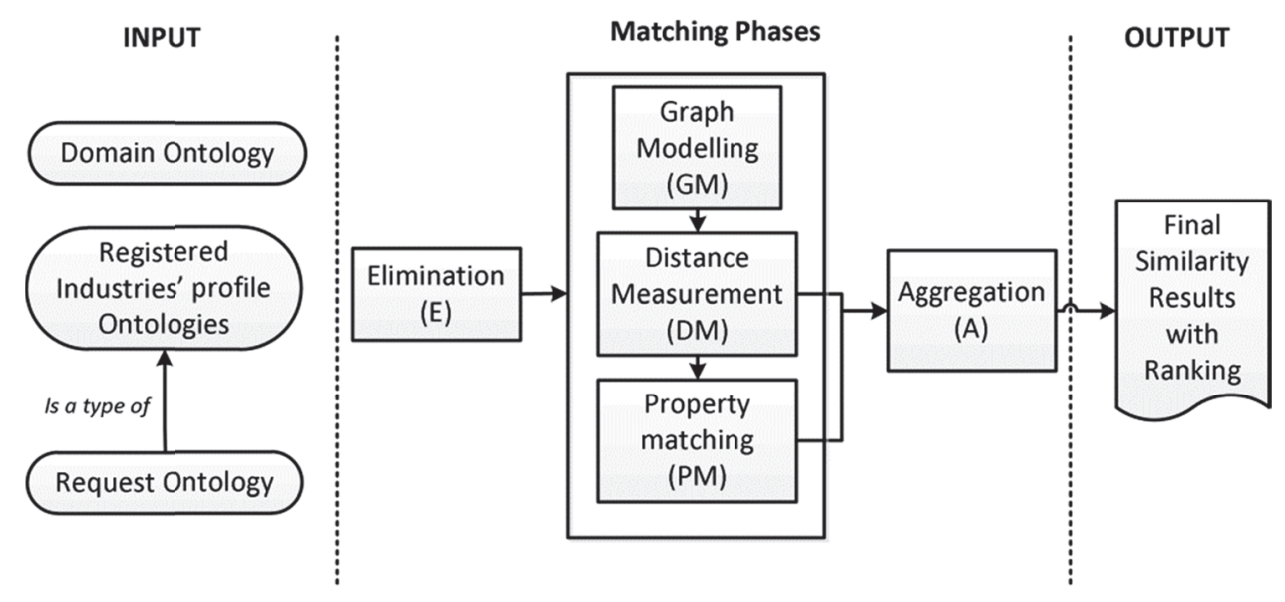

Figure 10 Process of input - output matching

The process of eliminations is introduced to minimise redundant matching and hence to computationally speed up the process. Three categories of elimination are introduced: i) elimination based on requester role, ii) elimination based on the nature of the resource in terms of hazardousness, and iii) elimination 
based on availability of resources. Requester role is defined by belonging to either the group of resource providers $x$ or to the group of solution providers $y$. All instances belonging to the requester's group and classes not disjoint by the requester are eliminated from matching. In the current ontology design disjoint classes indicate that their instances do not have technological relevance with each other. Considering the fact that different user roles are also defined by disjoint classes in the ontology, every industry which is capable of both providing and processing a resource will have to define two separate profiles under each role. Additionally, if the requester provides or askes for a hazardous material, all instances which are not categorised as hazardous are eliminated. Availability, which is characterised by properties isValidFrom and isValidTo, is also used in the elimination process as participants the availability of which do not overlap are eliminated. More precisely, availability is measured by the time overlap between the requester and matched instances, as demonstrated in Figure 5. For the availability period of the requester $s_{R}$ defined by the property $p_{R, k}=\left(P_{R, k}^{\text {start }}, P_{R, k}^{e n d}\right), p_{R, k} \in P_{k}^{n_{P}}$ and the availability period of matched instances $s_{j}$ defined by properties $p_{j, k}=\left(P_{j, k}^{\text {start }}, P_{j, k}^{e n d}\right), p_{j, k} \in P_{k}^{n_{P}}$, the overlap period $T_{o}$ is calculated as

$$
T_{o}=p_{R, k} \cap p_{j, m}=\min \left(P_{R, k}^{e n d}, P_{j, \mathrm{k}}^{e n d}\right)-\max \left(P_{R, k}^{\text {start }}, P_{j, \mathrm{j}}^{\text {start }}\right)
$$

The elimination is then based on the rule

$$
\text { IF } \begin{cases}T_{o} \leq 0 & \text { eliminate the match } \\ T_{o}>0 & \text { match }\end{cases}
$$

To account for both tacit and explicit knowledge in the process of matching, the tacit part of semantic similarity is quantified by the distance measurement between respective concepts along subsumption and relevant relationships (equation (13)), whereas explicit part of semantic similarity is quantified through the vector similarity calculation (equation (11)) of respective properties. Graph representation of the ontology is a prerequisite for both of them.

Various methods exist for determining graph model of the ontology such as those based on bipartite graphs accounting for the subsumption $H_{C}$ (Melnik et al. 2002) or combination of subsumption $H_{C}$ (Hu et al. 2005) and relationships hierarchy $R^{C}$ (Tous and Delgado 2006). We propose ontology graph models which are extension of bipartite graphs accounting for both relationships hierarchy $R^{C}$ and respective restrictions $f_{D}$. With this approach the ontology graphs are represented as a 3-tuple $G=\langle V, E, W\rangle$ :

$$
G= \begin{cases}V=\left\{H^{I}\right\} & \text { the set of vertices } \\ E=\left\{H_{C}, R^{C}, f_{D}\right\} & \text { the set of edges } \\ W=\left\{w_{i}\left(E_{i}\right) \mid w_{i} \in \mathbb{R}\right\} & \text { the weight assigned to each edge }\end{cases}
$$

We use only the relationships with a strong relevance to the domain of IS needed to infer tacit knowledge from the ontology:

$$
\begin{aligned}
& R_{\text {edge }}^{C}=\{\text { canProcess, canBeProcessedBy, hasApplicationIn, } \\
& \text { canUse, hasComposite, isCompositeOf }\} \mid R_{\text {edge }}^{C} \subseteq R^{C}
\end{aligned}
$$

as, along with is-a subsumption relationship, presented in Table 6. The equation (27) is modelled as an edge between domain class $S_{i}^{D}$ and $S_{j}^{R}$. For example, considering restrictions $f_{D}$ and $f_{R}$ presenting someValuesFrom on relationship $R_{i}^{C}=$ hasComposite between two concepts $S_{i}^{D}=$ EWC120103 (waste produced by non-ferrous metal turning and filing process) and $S_{j}^{R}=$ Aluminium, this restriction establishes semantic relevance and hence is modelled as edge between the two concepts, as shown in Figure 11. 


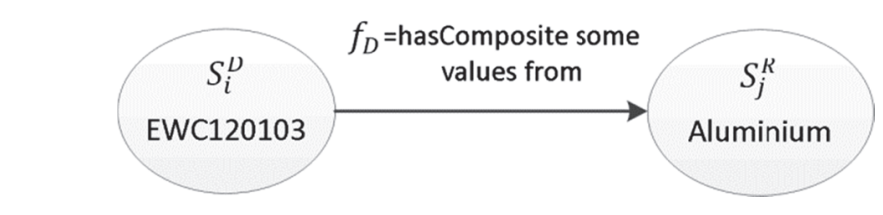

Figure 11 Edge modelling based on restriction on the property

To amplify semantic in IS sense and to better reflect IS practice, weighting is introduced such that stronger links in ontology graphs are given lower weights: relationships between equivalent classes have a minimum weight of 0 . Properties and their inverse properties have the same weights. Restrictions on properties have the same weights as the properties themselves, as demonstrated in Table 6.

Table 6 Semantic weighting of relationshi
\begin{tabular}{l|c} 
Edge & weight \\
\hline Subsumption(is-a) & 0.5 \\
equivalency & 0 \\
canProcess & 0.9 \\
canBeProcessedBy & 0.9 \\
hasApplicationIn & 0.7 \\
canUse & 0.7 \\
hasComposite & 0.6 \\
isCompositeOf & 0.6 \\
\hline
\end{tabular}

From practical point of view, the ontology graphs can be represented in matrix form:

$$
M=\left(\begin{array}{ccccc}
0 & m_{1,2} & m_{1,3} & \cdots & m_{1, n} \\
m_{2,1} & 0 & m_{2,3} & \cdots & m_{2, n} \\
m_{3,1} & m_{3,2} & 0 & \cdots & m_{3, n} \\
\vdots & \vdots & \vdots & \ddots & \text { inf } \\
m_{m, 1} & m_{m, 2} & m_{m, 3} & \cdots & 0
\end{array}\right)
$$

Here, the matrix elements $m_{i, j}$ represent weighted distance between each pair of graph nodes as defined in Table 6. From the ontology graph model, the distance $\delta\left(S_{i}^{I}, S_{C}^{I}\right)$ between two classes (equation (13)) is calculated as the dissimilarity function satisfying the definiteness and triangular inequality:

$$
\begin{array}{lll}
\forall S_{i}^{I}, S_{j}^{I} \in S, \delta\left(S_{i}^{I}, S_{j}^{I}\right)=0 \text { if and only if } S_{i}^{I}=S_{j}^{I} & \text { definiteness } \\
\forall S_{i}^{I}, S_{j}^{I}, S_{k}^{I}, \in S, \delta\left(S_{i}^{I}, S_{j}^{I}\right)+\delta\left(S_{j}^{I}, S_{k}^{I}\right) \geq \delta\left(S_{i}^{I}, S_{k}^{I}\right) & \text { triangular inequality }
\end{array}
$$

The distance similarity measure $h_{k}^{C}$, as in equation (13), is calculated as the shortest distance between two nodes in the graph

$$
h_{k}^{C}=\left(1-\delta^{\prime}\right) * 100
$$

where $\delta^{\prime}$ is normalised dissimilarity between two nodes. The normalisation is performed against the longest logical path between the two nodes in the graph. In practical terms, this process assures for technological relevance between two matched participants.

The property similarity between requester and user instances is used for I/O matching based on the explicit knowledge captured by the ontology. Four properties are used for property similarity calculation and they are specified in Table 7. In the present implementation we calculate property similarity from vector similarity, which combines cosine similarity $h_{k}^{V, c}$ with Euclidean similarity $h_{k}^{V, E}$ as a mean average $\left(h_{k}^{V}=\left(h_{k}^{V, c}+h_{k}^{V, E}\right) / 2\right)$. As such, the absence of a property value, normally replaced by 0 , does not penalise calculated similarity more than semantically justifiable. 
Table 7 Properties used for property similarity calculation

\begin{tabular}{l|c|l}
\multicolumn{1}{c|}{ Property } & Value Type & \multicolumn{1}{|c}{ Description } \\
\hline $\begin{array}{l}\text { Quantity } \\
\text { Location }\end{array}$ & $\begin{array}{c}\text { Float } \\
\text { Longitude and } \\
\text { Latitude } \\
\text { Date }\end{array}$ & $\begin{array}{l}\text { The exact value used in the vector } \\
\text { Measured as geographical distance to the requester }\end{array}$ \\
Pattern of Supply & Predefined text & $\begin{array}{l}\text { Measured as the percentage of overlap with the } \\
\text { availability specified by the requester } \\
\text { Takes the value 1 for continuous and 100 for batch type of } \\
\text { supply }\end{array}$ \\
\hline
\end{tabular}

The cosine similarity $h_{k}^{V, c}$ is calculated as a cosine of angle between the request vector $\mathbf{p}_{r}$ and other user vectors $\mathbf{p}_{i}$

$$
h_{k}^{V, c}=\cos (\theta)=\left\{\frac{\mathbf{p}_{r} \cdot \mathbf{p}_{i}}{\left\|\mathbf{p}_{r}\right\|\left\|\mathbf{p}_{i}\right\|}\right\}_{i=1}^{N}=\left\{\frac{\sum_{i=1}^{N} p_{r, i} \times p_{i, i}}{\sqrt{\sum_{i=1}^{N}\left(p_{r, i}\right)^{2}} \times \sqrt{\sum_{i=1}^{N}\left(p_{i, i}\right)^{2}}}\right\}
$$

where $N=4$ as evident from Table 7. To relax the deviation introduced by the absence of a property, Euclidean similarity $h_{k}^{V, E}$ is introduced as

$$
h_{k}^{V, E}=\sqrt{\sum_{i=1}^{N}\left(p_{r, i}-p_{i, i}\right)^{2}}
$$

The distance and property similarity are aggregated together as a fuzzy weighted average, which, according to eq. (14) is:

$$
h_{k}=\frac{\alpha h_{k}^{C}+\beta h_{k}^{V}}{(\alpha+\beta)}
$$

where $\alpha$ and $\beta$ are weighing parameters and in the current implementation we use $\alpha=0.6$ and $\beta=0.4$.

\section{Demonstration and Experimental Evaluation}

Two examples are used to demonstrate both the performance of designed ontology and matching algorithm and an optimised property decomposition to maximise environmental performance.

\subsection{Demonstration of Ontology and Matching Algorithm}

The first example illustrates the performance of the domain ontology and matching algorithm and their implementation as a service. The company names are illustrative with real data presented. Simplified examples with a limited number of six properties are used to make the experiment illustrative and purposeful. These include properties related to the type of $1 / 0$, quantity of available or requested resources, pattern of supply, availability period and location of the company. In reality, the number and type of properties and relationships used in the algorithm, from among the large number of them reflecting technological, economic and environmental conditions of IS, are selected to serve particular IS policy and local or otherwise set priorities and constraints.

Company 1 is a solution provider, an enterprise that produces chemicals for a wider market. Through the registration process Company 1 registers as a solution provider and provides other information essential for the matching process, as shown in Table 8. 
Table 8 Company 1 solution information

\begin{tabular}{|c|c|c|c|c|c|c|c|c|}
\hline \multirow[t]{2}{*}{ Company } & \multirow[t]{2}{*}{ Process } & \multirow{2}{*}{$\begin{array}{c}\text { Required } \\
\text { Resource } \\
\text { (input) }\end{array}$} & \multirow{2}{*}{$\begin{array}{c}\text { Quantity } \\
\text { (tonne/mo } \\
\text { nth) }\end{array}$} & \multirow{2}{*}{$\begin{array}{l}\text { Pattern } \\
\text { of } \\
\text { Supply }\end{array}$} & \multicolumn{2}{|c|}{$\begin{array}{c}\text { Availability } \\
\text { Period }\end{array}$} & \multicolumn{2}{|c|}{ Location } \\
\hline & & & & & From & To & Lat & Long \\
\hline $\begin{array}{c}\text { Company } \\
1\end{array}$ & $\begin{array}{l}\text { Anaerobic } \\
\text { Digestion }\end{array}$ & $\begin{array}{l}\text { Lignocellul } \\
\text { oses }\end{array}$ & 150 & $c^{*}$ & $\begin{array}{c}09 / 08 / \\
2012\end{array}$ & $\begin{array}{c}08 / 12 / \\
2015\end{array}$ & 38.339 & 23.61278 \\
\hline
\end{tabular}

${ }^{*} \mathrm{c}-$ continuous

After matching request by Company 1 has been placed, the matching process starts in stages. The elimination stage eliminates all the instances, the registered companies, from the process of matching which obviously do not meet the fundamental criteria or are instantiated in the domain ontology within classes which are not disjoint from class of the request and overlap of availability period $T_{o} \leq 0$. In the illustrative example all the companies belonging to the solution providers only are also eliminated. The remaining companies that could potentially provide matches with similarity $>0$ are listed in Table 9 .

Table 9 Profile of registered industries offering potential matches with Company 1

\begin{tabular}{|c|c|c|c|c|c|c|c|c|}
\hline \multirow{2}{*}{ ID } & \multirow{2}{*}{ Company } & \multirow{2}{*}{$\begin{array}{c}\text { Produced } \\
\text { Resource } \\
\text { type(output) }\end{array}$} & \multirow{2}{*}{ Quantity } & \multirow{2}{*}{$\begin{array}{l}\text { Supply } \\
\text { Pattern }\end{array}$} & \multicolumn{2}{|c|}{ Availability } & \multicolumn{2}{|c|}{ Location } \\
\hline & & & & & Valid From & Valid To & Lat & Long \\
\hline 27 & Company 3 & Wood & 230 & 2 & $14 / 11 / 2014$ & $14 / 12 / 2015$ & 38.325 & 23.600 \\
\hline 22 & Company 4 & MDF & 50 & 2 & 07/08/2013 & $17 / 10 / 2015$ & 38.326 & 23.581 \\
\hline 3 & Company 2 & EWC030308 & 90 & 2 & 04/07/2012 & 03/06/2016 & 38.345 & 23.631 \\
\hline 187 & Company 5 & Cardboard & 450 & 2 & 07/08/2013 & $17 / 12 / 2014$ & 38.329 & 23.612 \\
\hline 144 & Company 6 & EWC020103 & 80 & 1 & 07/08/2012 & $14 / 12 / 2015$ & 38.325 & 23.631 \\
\hline 44 & Company 7 & EWC020705 & 70 & 2 & 09/08/2012 & 09/09/2016 & 38.342 & 23.581 \\
\hline 1 & Company 8 & EWC030301 & 90 & 1 & 06/11/2012 & $05 / 07 / 2016$ & 38.345 & 23.611 \\
\hline 19 & Company 9 & Lignocellulosic & 200 & 1 & 04/07/2012 & $17 / 10 / 2015$ & 38.378 & 23.631 \\
\hline
\end{tabular}

The next stage of matching includes matching the 1/O type from the properties related to required and produced resources which are referenced by the class/concepts the instance is attached to. The distance measurement similarity $h_{k}^{C}$ between requesting and other instances in the domain ontology is used as the measure of input/output matching. The excerpt of the domain ontology explaining the input - output matching between Company 1 and Company 2 is shown in Figure 12. 




Figure 12 Excerpt of the domain ontology used for input output matching

The shortest distance between the two concepts EWC030308 and LingocellulosicProducts are recovered by two edges through the concept Paper with the total value 1.1, as shown in Figure 13 and using the values defined in Table 6, which normalised to the longest path between them in the ontology gives

$$
\delta^{\prime}=\frac{\text { distance }}{D_{c}}=\frac{1.1}{4.5}=0.24
$$

and then the similarity as

$$
h_{k}^{C}=(1-0.24)=0.76
$$

The semantic of calculated similarity measure (35) is that lignocellulosic products also include paper and hence providing the match between requested lignocelluloses and EWC 030308 with the similarity of $76 \%$.

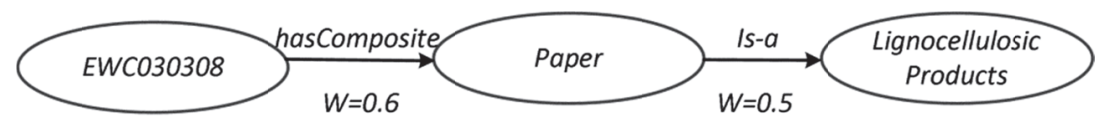

Figure 13 Distance measurement example

The property similarity is calculated between the two companies based on the set of general properties for the company $P_{i}^{n_{P}}=$ (Location, Resource) and the set of properties characterising the resources they request or have $P_{j}^{n_{P}}$ (=(Quantity $, \underline{\text { Availability }}, \underline{\text { PatternofSupply) }}$ ) with those underlined needed for the property matching. A set of four numerical properties $P_{i}^{N}=P_{i}^{4}$ is calculated which includes 


$$
P_{i}^{4}=\{v(\text { Availability), } v(\text { Quantity }), v(\text { PatternofSupply), } v(\text { Location })\} .
$$

The Availability is calculated from equation (24) as the overlap period $T_{o}=1216$ days which results in $100 \%$ overlap between the two companies. The property Location is calculated as the distance between the two companies using Haversine formula which in normalised form gives $L_{\text {distance }}=1.7$. Hence, $P_{i}^{4}$ is modelled as 4-dimensional vectors which for Company 1 and Company 2 are $\mathbf{p}_{1}=$ $(33.33,100,100,0)$ and $\mathbf{p}_{2}=(20,100,100,37.36)$, respectively giving the cosine similarity (equation (27))

$$
h_{k}^{V, c}=0.937
$$

and Euclidean similarity (from equation (32))

$$
h_{k}^{V, E}=0.709
$$

which combined together as property similarity between the two companies calculate as

$$
h_{k}^{V}=\left(h_{k}^{V, c}+h_{k}^{V, E}\right) / 2=0.818
$$

The aggregated similarity between the two companies Company 1 and Company 2 from equation (14) is

$$
h_{k}=\frac{\alpha h_{k}^{C}+\beta h_{k}^{V}}{(\alpha+\beta)}=(0.6 \times 0.76)+\frac{(0.4 \times 0.818)}{0.6+0.4} \times 100=78 \%
$$

where $\alpha=0.6$ and $\beta=0.4$ reflecting that the types of resource of the companies has a greater effect on the possibility of the establishment of a synergy.

In retrospect, from the whole process and based on the $78 \%$ similarity between the companies based on the information they provided (Table 8 and Table 9), it is evident that Company 1 has a fair chance of processing at least part of the waste produced by Company 2. The two companies also reside in a comparatively close proximity. The one notable aspect which lowers the similarity result is the fact that Company 2 can supply Company 1 with only $60 \%$ of its required resource. In practical terms, however, Company 1 still has a possibility to supplement remaining capacities through other synergies.

Following the same procedure, the matches with other companies are determined and the final results are summarised in Table 10.

\section{Table 10 Complete set of results}

\begin{tabular}{lcccc} 
Company & $\begin{array}{c}\text { Semantic } \\
\text { distance } \\
\text { similarity }\end{array}$ & $\begin{array}{c}\text { Average } \\
\text { property } \\
\text { similarity }\end{array}$ & $\begin{array}{c}\text { Aggregated } \\
\text { results }\end{array}$ & $\begin{array}{c}\text { Similarity } \\
\text { percentage }\end{array}$ \\
\hline Company 9 & 1 & 0.487325 & 0.79493016 & $79 \%$ \\
Company 3 & 0.89 & 0.221338 & 0.622535374 & $62 \%$ \\
Company 8 & 0.87 & 0.909041 & 0.885616538 & $89 \%$ \\
Company 5 & 0.78 & 0.239821 & 0.56392828 & $56 \%$ \\
Company 2 & 0.76 & 0.818824 & 0.783529759 & $78 \%$ \\
Company 4 & 0.67 & 0.283113 & 0.51524522 & $52 \%$ \\
Company 6 & 0.44 & 0.764722 & 0.569888781 & $57 \%$ \\
Company 7 & 0.44 & 0.398214 & 0.423285785 & $42 \%$ \\
\hline
\end{tabular}

As described in Section 2.5, Company 7 is not to be considered for optimisation given the default value for similarity threshold is $h_{k}^{\text {tres }}=0.5$. 


\subsection{Demonstration of Environmental Optimisation}

The second example, a case study set in the Viotia region in Greece, is used to demonstrate the property decomposition and respective optimisation to maximise environmental performance. Again, simplified examples with a limited number of properties are used to make the experiment illustrative and purposeful. Also, examples use real-life data with the names of the companies replaced for confidentiality reasons.

User 9 is a resource consumer which requires polypropylene for the production of flexible packaging materials. The company is investigating a possibility to utilise extra sources of input materials for a three year period, with the full set of requirements given in Table 11, the requirements provided during the registration and used for matching.

Table 11 User 9 registration information

\begin{tabular}{|c|c|c|c|c|c|c|c|c|}
\hline \multirow{2}{*}{ ID } & \multirow{2}{*}{$\begin{array}{l}\text { User } \\
\text { Type }\end{array}$} & \multirow{2}{*}{$\begin{array}{l}\text { Resource } \\
\text { Input }\end{array}$} & \multirow{2}{*}{$\begin{array}{c}\text { Input } \\
\text { Quantity }\end{array}$} & \multicolumn{2}{|c|}{ Location } & \multirow{2}{*}{ Valid from } & \multirow{2}{*}{ Valid to } & \multirow{2}{*}{$\begin{array}{l}\text { Pattern of } \\
\text { Supply }\end{array}$} \\
\hline & & & & Lat & Lon & & & \\
\hline 9 & $\mathrm{RC}^{*}$ & Polypropylene & 810.00 & 22.9165 & 38.6466 & $01 / 06 / 2013$ & $01 / 06 / 2015$ & $c^{*}$ \\
\hline
\end{tabular}

After the matching request has been placed, the matching process described in Section 3.2 is initiated. All registered companies which do not fulfil criteria specified in Section 3.2 are eliminated during the elimination stage of $\mathrm{I} / \mathrm{O}$ matching. The companies that offer potential matches with there is properties are presented in Table 12.

Table 12 Registration details of potential matches

\begin{tabular}{|c|c|c|c|c|c|c|c|c|c|}
\hline \multirow{2}{*}{ ID } & \multirow{2}{*}{$\begin{array}{l}\text { User } \\
\text { Type }\end{array}$} & \multirow{2}{*}{$\begin{array}{c}\text { Resource } \\
\text { Output }\end{array}$} & \multirow{2}{*}{$\begin{array}{c}\text { Resource } \\
\text { Input }\end{array}$} & \multirow{2}{*}{$\begin{array}{l}\text { Output } \\
\text { Quantity }\end{array}$} & \multicolumn{2}{|c|}{ Location } & \multirow{2}{*}{ Valid from } & \multirow{2}{*}{ Valid to } & \multirow{2}{*}{$\begin{array}{c}\text { Pattern of } \\
\text { Supply }\end{array}$} \\
\hline & & & & & Lat & Lon & & & \\
\hline 2 & $\mathrm{SP}^{*}$ & Polypropylene & Propylene & 830.00 & 22.8563 & 38.5251 & $01 / 06 / 2013$ & $01 / 01 / 2015$ & $b^{*}$ \\
\hline 5 & $\mathrm{RP}^{*}$ & PP Scrap Bags & - & 600.00 & 22.938 & 38.4323 & $10 / 09 / 2013$ & $01 / 07 / 2014$ & $\mathrm{c}^{*}$ \\
\hline 6 & $\mathrm{RP}^{*}$ & Propylene & - & 550.00 & 22.8296 & 38.5188 & $01 / 01 / 2013$ & $01 / 01 / 2015$ & $\mathrm{c}^{*}$ \\
\hline 1 & $\mathrm{RP}^{*}$ & Propylene & - & 850.00 & 22.8923 & 38.5251 & $01 / 06 / 2013$ & $01 / 01 / 2015$ & $b^{*}$ \\
\hline
\end{tabular}

${ }^{*} \mathrm{RC}$ - resource consumer, SP - solution provider, $\mathrm{b}$ - batch, $\mathrm{c}$ - continuous

In addition, the information used to calculate environmental performance for all identified matches, as well as the requestor, extracted from respective properties in the ontology, are presented in Table 13.

Table 13 Environmental information

\begin{tabular}{llccccccc}
\hline ID & $\begin{array}{c}\text { User } \\
\text { Type }\end{array}$ & Resource & $\begin{array}{c}\text { Embodied } \\
\text { Carbon } \\
\left(\mathrm{kgCO}_{2} / \mathrm{kg}\right)\end{array}$ & $\begin{array}{c}\text { Feedstock } \\
\text { Price } \\
(\mathrm{f} / \text { tonne })\end{array}$ & $\begin{array}{c}\text { Resource } \\
\text { Price } \\
(\mathrm{f} / \text { tonne })\end{array}$ & $\begin{array}{c}\text { Disposal } \\
\text { Cost } \\
(\mathrm{f} / \text { tonne })\end{array}$ & $\begin{array}{c}\text { Landfill } \\
\text { Tax } \\
(\mathrm{f} / \text { tonne })\end{array}$ & $\begin{array}{c}\text { Transportation } \\
\left(\mathrm{kgCO} / \mathrm{km}^{*} \text { tonne }\right)\end{array}$ \\
\hline $\mathbf{9}$ & $\mathrm{RC}$ & Polypropylene & - & $\mathbf{1 8 1 0}$ & - & - & - & - \\
\hline $\mathbf{2}$ & $\mathrm{SP}^{*}$ & Polypropylene & 3.9 & 1810 & - & 30 & 43 & 0.906 \\
\hline 5 & $\mathrm{RP}^{*}$ & PP Scrap Bags & 1.8 & - & 600 & 30 & 43 & 0.906 \\
\hline $\mathbf{6}$ & $\mathrm{RP}^{*}$ & Propylene & 1.35 & 1000 & 845 & 43 & 43 & 0.906 \\
\hline $\mathbf{1}$ & $\mathrm{RP}^{*}$ & Propylene & 1.35 & - & - & 43 & 43 & 0.906 \\
\hline
\end{tabular}

It becomes apparent from Table 12 and Table 13 that the $1 / 0$ matching without the property decomposition offer three possible solutions, as illustrated in Figure 14; solution (1) which includes companies 1-2-9, solution (2) which includes companies 6-2-9, and solution (3) which includes companies 59 , all with the final product polypropylene, as requested by the requester 9 . 




Figure 14 IS network solution without property decomposition

The (aggregated) similarity for each network option (1), (2) and (3) in Figure 14 is calculated according to eq. (14) and the environmental effects according to eq. (17) yielding the results given in Table 14. In this case the quantities and the availability periods are used only to maximum satisfaction of the follow-on stage, which is the fundamental principle of the semantic matching. Also, it is obvious in this example that the backward chaining process is used because the requester is a resource consumer company with defined and targeted output, polypropylene in this case, as well as that the combined (1), (2) and (3) solution is the full solution because it includes all the partners satisfying the technological and IS logic, as defined by the decomposition principle vi) in Section 2.5 .

Table 14 Similarity results and environmental effects

\begin{tabular}{cccc}
\hline $\begin{array}{c}\text { Synergy } \\
\text { No. }\end{array}$ & IS network & $\begin{array}{c}\text { Similarity } \\
\boldsymbol{h}_{\boldsymbol{k}}\end{array}$ & $\begin{array}{c}\text { Environmental } \\
\text { effects } \boldsymbol{E} \boldsymbol{N} \boldsymbol{V} \boldsymbol{I}\end{array}$ \\
\hline$(1)$ & $1-2-9$ & 0.8351 & 782.17 \\
$(2)$ & $6-2-9$ & 0.8297 & 294.39 \\
$(3)$ & $5-9$ & 0.9017 & 129.90 \\
\hline
\end{tabular}

The next step involves inclusion of the optimized decomposition of properties related to quantities, hasquanity, and to availability, has Availability calculated using eq. (20), for which the optimisation model for all the partners identified by semantic matching, namely partners (1), (2), (5) and (6), along with the requester (9), is defined as 


$$
\begin{aligned}
& \text { maximise } \quad E N V I=Q_{9,2} *\left(D C_{9,2}+L T\right)-\left(Q_{9,2} * E C_{9,2} * C O_{2}^{P}\right)-\left(Q_{9,2} * T F_{9,2} * \text { distance }_{9,2} * C O_{2}^{P}\right)+C_{9} *\left(F P_{9,2}-R P_{9,2}\right) \\
& +Q_{9,5} *\left(D C_{9,5}+L T\right)-\left(Q_{9,5} * E C_{9,5} * C O_{2}^{P}\right)-\left(Q_{9,5} * T F_{9,5} * \text { distance }_{9,5} * C O_{2}^{P}\right)+C_{9} *\left(F P_{9,5}-R P_{9,5}\right) \\
& +Q_{2,1} *\left(D C_{2,1}+L T\right)-\left(Q_{2,1} * E C_{2,1} * C O_{2}^{P}\right)-\left(Q_{2,1} * T F_{2,1} * \text { distance }_{2,1} * C O_{2}^{P}\right)+C_{2} *\left(F P_{2,1}-R P_{2,1}\right) \\
& \text { s.t. } \\
& +Q_{2,6} *\left(D C_{2,6}+L T\right)-\left(Q_{2,6} * E C_{2,6} * C O_{2}^{P}\right)-\left(Q_{2,6} * T F_{2,6} * \text { distance }_{2,6} * C O_{2}^{P}\right)+C_{2} *\left(F P_{2,6}-R P_{2,6}\right) \\
& Q_{9,5}+Q_{9,2} \leq C_{9} \\
& Q_{2,6}+Q_{2,1} \leq C_{2} \\
& Q_{9,5} * \text { existence }_{9,5}+Q_{9,2} * \text { existence }_{9,2}=C_{9} \\
& \mathrm{EQ}_{9} \leq \mathrm{EQ}_{2}+\mathrm{EQ}_{5} \\
& E T_{9} \geq \text { Overlap }_{9,2} \\
& E T_{9} \geq \text { Overlap }_{9,5} \\
& E T_{2} \geq \text { Overlap }_{2,1} \\
& E T_{2} \geq \text { Overlap }_{2,6} \\
& Q_{i, j}, E T_{k} \geq 0 \\
& \text { existence }_{i, j} \in\{0,1\}
\end{aligned}
$$

\begin{tabular}{|c|c|c|c|c|c|}
\hline $\begin{array}{c}\text { Synergy } \\
\text { No. } \\
\end{array}$ & IS network & $\begin{array}{c}\text { Similarity } \\
\boldsymbol{h}_{\boldsymbol{k}}\end{array}$ & $\begin{array}{c}\text { Environmental } \\
\text { effects } E N V I\end{array}$ & $\begin{array}{c}\text { Normalised } \\
\text { ENVI } I_{i, j}^{\text {normalised }} \\
\end{array}$ & $\begin{array}{l}\text { Score } \\
M_{n w} \\
\end{array}$ \\
\hline (1) & $1-2-9$ & 0.8351 & 782.17 & 0.2212 & 0.4053 \\
\hline (2) & $6-2-9$ & 0.8297 & 294.39 & 0.0 & 0.2489 \\
\hline (3) & $5-9$ & 0.9017 & 129.90 & 0.8770 & 0.8844 \\
\hline Optimised & $6-2-5-9$ & 0.883 & 873.662 & 1.0 & 0.9648 \\
\hline 1 & & & 2 & & \\
\hline & & & & & 9 \\
\hline 6 & & & 5 & & \\
\hline
\end{tabular}

where variable $Q_{i, j}$ refers to optimised quantities, variable $E T_{k}$ refers to optimised availability, parameter distance $_{i, j}$ is the distance between partners, and all other parameters are defined in Table 2. The concomitant results are summarised in Table 15 with graphical illustration for optimal quantities in Figure 15. Here in Table 15, the aggregated score $M_{n w}$ is calculated according to the eq. (23). In addition, optimised availability periods are shown in Figure 16. It becomes apparent that availability periods are the same as the original ones in Table 11 and Table 12 because of the full overlap at each of the two matching stages, as well as that the requester availability is only partially satisfied with additional 5 months to spare. It also becomes apparent from presented results that the optimised solution includes participants 6-2-5-9 and that it is a sub-solution of the 1-2-5-6-9 full solution presented in Figure 14. Although the aggregated semantic similarity for the optimised solution is not the best, only scores value of 0.883 , that solution provides the best environmental effects $(E N V I=873.662)$ and hence gains the best aggregated score of $M_{n w}=0.9648$.

Table 15 A full set of solutions

Figure 15 The optimised solution

In addition from a detailed analysis of the results it becomes apparent that the exchanged quantities are optimised to maximise the environmental performance of the network. For example, Company 1 is disregarded probably because of the long distance $(9.34 \mathrm{~km})$ from Company 2 in comparison to Company 6 $(2.51 \mathrm{~km})$. Also, the optimisation process suggests the use of more material from Company 5 offering scrap which in turn affects environmental performance more. 


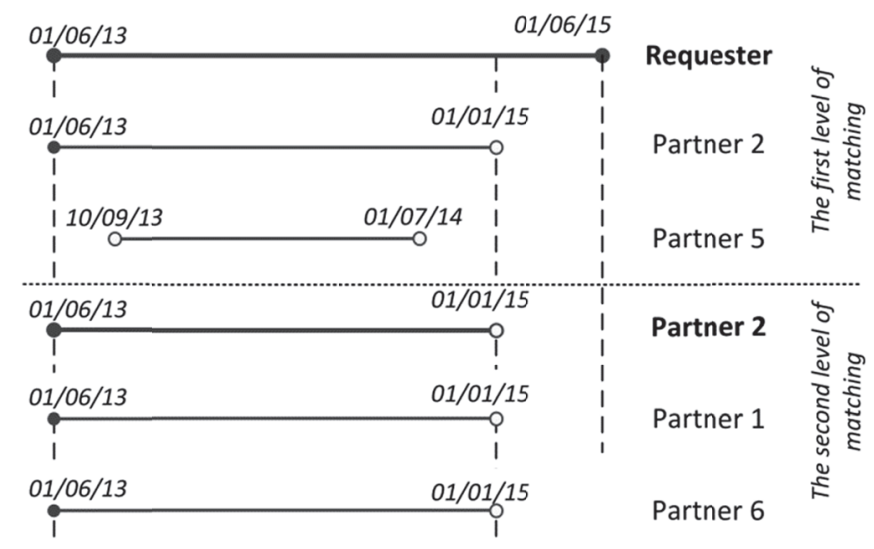

Figure 16 The availability overlap at two matching stages

As a matter of illustration, the geographical representation of the optimised IS networks is demonstrated in Figure 17.



Figure 17 Geographical representation of the optimised solution

\section{Conclusion}

A complete semantic algorithm for IS networking is presented. The algorithm introduces ontology modelling of the knowledge in the domain of IS. Designed with four levels of abstraction, ontology embeds the tacit knowledge acquired from IS experts, including resource and solution classifications and characterisation, role of participants, as well as the operational aspects of IS. The designed ontology also enables acquisition of explicit knowledge through the process of increasing the population, the ontology instantiation. The input - output matching is also presented. Individuals are matched on their semantic and explicit relevance. Semantic relevance, the semantic similarity, is quantified by the distance measured between respective concepts in the ontology. Explicit relevance is calculated from the vector similarity of instance properties. Aggregated similarity is used to rank the matches by the IS relevance. Innovative networks are formed by enabling decomposition of properties, namely properties associates with the quantities and resource availability. The process of decomposition, as presented, is optimised to maximise environmental performance. The proposed algorithm is implemented as a web service and its use demonstrated by several case studies and with a step-by-step valorisation and justification by respective semantic. 


\section{Acknowledgement}

The authors would like to acknowledge European financial instrument for the Environment (LIFE+), LIFE09 ENV/GR/000300, financial support. Special thanks goes to Avco Systems Itd. U.K. for developing of the service into a web service available for use.

\section{Appendix A: The top four level of the IS domain ontology}

\begin{tabular}{|c|c|}
\hline Concepts & Description \\
\hline Role & The participants of IS process. \\
\hline ResourceProducer & Participants who have a resource available. \\
\hline SolutionProvider & Participants who have some solution available. \\
\hline Resource & Resource in IS contains materials, wastes, water, energy etc. \\
\hline ResourceByType & Resources that can be naturally classified by their type \\
\hline Materials & The substance or substances out of which a thing is or can be made \\
\hline Polymers & $\begin{array}{l}\text { This concept ranges from synthetic plastics and elastomers to natural } \\
\text { biopolymers. }\end{array}$ \\
\hline Metals & $\begin{array}{l}\text { Solid materials which are typically hard, shiny, malleable, fusible, and } \\
\text { ductile, with good electrical and thermal conductivity. Includes ferrous } \\
\text { and non-ferrous metals and alloys. }\end{array}$ \\
\hline Ceramics & $\begin{array}{l}\text { Inorganic, non-metallic materials generally made using clay and other } \\
\text { earthen materials through heat and cooling. }\end{array}$ \\
\hline Chemicals & Any material with a definite chemical composition. \\
\hline Minerals & $\begin{array}{l}\text { A mineral is a naturally occurring inorganic solid, with a definite chemical } \\
\text { composition, and an ordered atomic arrangement. }\end{array}$ \\
\hline Composites & $\begin{array}{l}\text { Naturally occurring or engineered materials made from two or more } \\
\text { constituents. }\end{array}$ \\
\hline OrganicMatter & Matter that comes from a once living organism such as plants and animals. \\
\hline Rocks & $\begin{array}{l}\text { The solid mineral materials forming part of the surface of the earth and } \\
\text { other planets. }\end{array}$ \\
\hline Energy & Usable heat or power. \\
\hline Electricity & $\begin{array}{l}\text { The supply of electric current to a house or other building for heating, } \\
\text { lighting, or powering appliances. }\end{array}$ \\
\hline Heat & $\begin{array}{l}\text { The transfer of energy from one body to another as a result of a difference } \\
\text { in temperature or a change in phase. }\end{array}$ \\
\hline Water & A clear, colorless, odorless, and tasteless liquid, $\mathrm{H}_{2} \mathrm{O}$. \\
\hline ResourceBySource(EWC) & Based on EWC - waste classification based on the source process. \\
\hline Products & The totality of goods that can be made available by industries. \\
\hline EnergyProducts & Goods that can be used for the generation of energy. \\
\hline Biomass & Organic matter used as a fuel. \\
\hline Biofuels & Fuels derived directly from living matter. \\
\hline Coal & $\begin{array}{l}\text { A combustible black or dark brown rock consisting mainly of carbonized } \\
\text { plant matter, found mainly in underground deposits and widely used as } \\
\text { fuel. }\end{array}$ \\
\hline NaturalGas & Flammable gas, consisting largely of methane and other hydrocarbons. \\
\hline Oil & A viscous liquid derived from petroleum. \\
\hline OilShale & Fine-grained sedimentary rock from which oil can be extracted \\
\hline Peat & $\begin{array}{l}\text { A brown, soil-like material, consisting of partly decomposed vegetable } \\
\text { matter. }\end{array}$ \\
\hline MaterialProducts & All other goods, not included in class EnergyProducts. \\
\hline ResourceByCharacteristic & Resources classified based on important physical or chemical properties \\
\hline
\end{tabular}


BiodegradableResource

Technology

TechnologyByType

TechnologyBylndustry

TechnologyByInput

TechnologyByCharacteristic

Attributes

geo:SpatialThing

Location

NACE

QuantityType

PatternOfSupply

Region

UnitOfMeasurement
Resources that are capable of decaying through the action of living organisms.

Any technological process that can convert an input to a different output under certain circumstances and with a specific result.

Technologies classified by their type.

Technologies classified based on the industry they can be applied in

Technologies classified based on their input.

Technologies classified based on important physical or chemical requirements they have.

Information used to describe and define all the concepts of the ontology.

Imported concept which links to the latitude and longitude information.

Linked to the above concept, the lat and long of the participant.

Statistical classification of economic activities

in the European Community.

The physical form of a resource.

The pattern that a resource is produced or required.

Linked to location, embracing the local aspect of IS.

Units of measurement.

\section{Appendix B: Description of used relationships $R_{i}^{C}$}

\begin{tabular}{|c|c|}
\hline Relationship $R_{i}^{C}$ & Description \\
\hline geo:location & $\begin{array}{l}\text { Term of a basic RDF vocabulary that provides the Semantic Web } \\
\text { community with a namespace for representing lat(itude), } \\
\text { long(itude) and other information about spatially-located things, } \\
\text { using WGS84 as a reference datum. }\end{array}$ \\
\hline belongsTolndustry & $\begin{array}{l}\text { Link between participants and the industry sector code (NACE) } \\
\text { they belong to. }\end{array}$ \\
\hline hasResource & $\begin{array}{l}\text { Link between a resource provider and the type of resource they } \\
\text { have available. }\end{array}$ \\
\hline hasTechnology & $\begin{array}{l}\text { Link between a solution provider and the type of solution they } \\
\text { have available. }\end{array}$ \\
\hline hasPatternOfSupply & $\begin{array}{l}\text { Links resources to the PatternOfSupply attribute concept. The } \\
\text { concept is about the pattern of the demand or availability } \\
\text { (Continuous, Batch). }\end{array}$ \\
\hline hasApplicationIn & $\begin{array}{l}\text { Link between resources and industry sectors for the integration of } \\
\text { tacit knowledge about the use of resources in different industries. }\end{array}$ \\
\hline canUse & $\begin{array}{l}\text { Inverse relation of hasApplicationln, used for intelligent } \\
\text { recommendations. }\end{array}$ \\
\hline hasQuantityType & $\begin{array}{l}\text { Links resources to the QuantityType attribute concept. The } \\
\text { concept is about the physical form of the resource (Solid, Liquid } \\
\text { etc.). }\end{array}$ \\
\hline hasUnitOfMeasurement & $\begin{array}{l}\text { Links resources and solutions to the UnitOfMeasurement attribute } \\
\text { concept. The concept is about the unit of measurement of the } \\
\text { resource ( } \mathrm{Kg} \text {,Tonnes etc.). }\end{array}$ \\
\hline hasComposie & $\begin{array}{l}\text { Relation used to provide information about the composition of } \\
\text { products and waste types. }\end{array}$ \\
\hline isCompositeOf & The inverse of the above. \\
\hline hasInput & Relation used to link solutions to their inputs. \\
\hline canProcess & Relation used to link solutions to their main inputs. \\
\hline needsWater & Relation used to link solutions to their water inputs. \\
\hline needsEnergy & Relation used to link solutions to their energy inputs. \\
\hline
\end{tabular}




\author{
canBeprocessedBy \\ hasOutput \\ hasProduct \\ hasStorageMethod \\ hasDeliveryMethod \\ hasInterval
}

Inverse relation of canProcess. Used for tacit knowledge modelling for resource processing.

Relation used to link solutions to their outputs.

Relation used to link solutions to their products.

Relation used to link resources to the storage methods used for their storage.

Relation used to link resources to the current method of delivery for resources.

Links resources to the interval related to the amount of resource produced.

\section{Appendix C: List of Properties}

\begin{tabular}{l|l}
\multicolumn{1}{c|}{\begin{tabular}{c}
\multicolumn{1}{c}{ Property $P_{i}^{N}$} \\
$\begin{array}{l}\text { confidentialityFlag } \\
\text { hasQuantity } \\
\text { hasProcessingPrice }\end{array}$
\end{tabular}} & $\begin{array}{l}\text { Description } \\
\text { The amount of resource available or required. } \\
\text { Addressing solution providers - the cost for the resource currently in use. }\end{array}$ \\
$\begin{array}{l}\text { hasAnnualCost } \\
\text { isValidFrom } \\
\text { isValidTo } \\
\text { hasName } \\
\text { isBiodegradable } \\
\text { isHazardous }\end{array}$ & $\begin{array}{l}\text { Addressing solution providers - the annual cost of a resource as feedstock. } \\
\text { The date the resource/solution becomes available. } \\
\text { The date the resource/solution stops being available. } \\
\text { Free text entry for the user to specify the name of the resource/solution. } \\
\text { Boolean property used to identify resources that are biodegradable. } \\
\text { Boolean property used to identify resources that are hazardous (contaminated } \\
\text { etc.) as defined in the European Waste Catalogue (EWC). } \\
\text { Boolean property used to identify whether the user can deliver the resource on } \\
\text { offer. } \\
\text { The amount of resource the user can store when requesting or producing a } \\
\text { resource. }\end{array}$ \\
hasStorageCapacity
\end{tabular}

\section{Notation}

$f_{D} \quad$ relationship restriction function on its domain

$f_{R}$ relationship restriction function on its range

$h_{i}$ h-metric

$h_{k}^{C}$ vector similarity measure

$h_{k}^{V}$ distance similarity measure vector similarity based on cosine algorithm vector similarity based on Euclidean algorithm

$H^{I} \quad$ set of classes

$i, j \quad$ general indices

$I_{i}^{I} \quad$ intension of a class

$M \quad$ matrix of a subsumption graph

$n_{C} \quad$ the total number of instances sharing common properties

$n_{I} \quad$ number of IS participants 


$\begin{array}{ll}n_{P} & \text { number of properties characterising all instances (individuals) } \\ n_{R} & \text { number of relationships organised in subsumption } \\ n_{T} & \text { number of solution (technology) providers } \\ n_{W} & \text { number of resource (waste) providers } \\ \mathbb{N} & \text { set of natural numbers } \\ N_{i}^{I} & \text { name of the class } \\ O & \text { ontology } \\ Q^{N} & \text { vector space of } n_{I} \text { vectors } \\ p_{i, j} & \text { property } \\ \mathbf{p}_{i} & \text { vector composed of N numerical properties } \\ P_{i}^{n_{P}} & \text { set of properties characterising instances } S_{i} \\ P_{i}^{N} & \text { set of numerical properties } \\ P_{R, k}^{s t a r t} & \text { property defining start of the availability period } \\ P_{R, k}^{e n d} & \text { property defining end of the availability period } \\ r_{i, j} & \text { relationship between two instances } \\ r_{i, j}^{-1} & \text { inverse instant relationship } \\ \mathbb{R} & \text { set of real numbers } \\ R^{C} & n_{R} \text {-dimensional relationship subsumption } \\ R_{i}^{C} & \text { class relationship } \\ R_{i}^{C} & \text { inverse class relationship of } R_{i}^{C} \\ R_{i}^{C} & \text { relationsip between two classes } \\ S_{i} & \text { an individual (instance) } \\ S & \text { set of all individuals, the IS participants } \\ S_{i}^{D} & \text { relationship domain class } \\ S_{i}^{I} & \text { ordered set representing a class } \\ S_{i}^{R} & \text { relationship range class } \\ T_{o} & \text { availability period overlap } \\ \alpha, \beta & \text { similarity weighting factors } \\ \delta_{i} & \text { the distance between classes } S_{i}^{I}\left(S_{j}^{I} \text { ) and another class } S_{C}^{I}\right. \\ & \end{array}$

\section{References}

Alberta, C. C. and H. G. Kevin (2008). Use of Industrial By-Products in Urban Transportation Infrastructure: argument for increased industrial symbiosis. Life Cycle Assessment VIII, Seattle, WA, USA, IEEE.

Cecelja, F., T. Raafat and N. Trokanas (2014a). eSymbiosis ontology. www.eSymbiosis.gr:

Cecelja, F., T. Raafat, N. Trokanas, S. .Innes, M. Smith, A. Yang, Y. Zorgios, A. Korkofygas and A.Kokossis (2014b). e-Symbiosis: technology-enabled support for industrial symbiosis targeting SMEs and innovation. Journal of Cleaner Production Online since 16 September 2014: pp 1. 
Chertow, M. (2000). Industrial Symbiosis: Literature and Taxonomy. Annu. Rev. Energy Environ. 25(1): pp 313-337.

Chertow, M. (2004). Industrial Symbiosis. Encyclopedia of Energy: pp 407-415.

Chertow, M. (2007). Uncovering Industrial Symbiosis. Journal of Industrial Ecology 11(1): pp 11-29.

Chertow, M. and J. Ehrenfeld (2012). Organizing Self-Organizing Systems - Toward a Theory of Industrial Symbiosis. Journal of Industrial Ecology 16(1): pp 13-27.

EC. (2002). "European Waste Catalogue: Commission Decision (EC) 2000/532 " Retrieved 14/06/2013, from http://ec.europa.eu/environment/waste/framework/list.htm.

EC (2010). Comission Regulation (EU) No 849/2010: Generation of Waste. Official Journal of the European Union 28.9.2010: pp 2-40.

Euzenat, J. and P. Shvaiko (2013). Ontology Matching, Springer.

Grant, G., T. Seager, G. Massard and L. Nies (2010). Information and Communication Technology for Industrial Symbiosis. Journal of Industrial Ecology 14(5): pp 740-753.

Hu, W., N. Jian, Y. Qu and Y. Wang (2005). A Graph Matching for Ontologies. K-Cap 2005 Workshop on Integrating Ontologies. Alberta, Canada: pp 43-50.

Jae-Yeon, W., K. Jung-Hoon, L. Sang-yoon and P. Hung-Suck (2006). Industrial Symbiosis as an Integrated Business/Environment Management Process: The Case of Ulsan Industrial Complex. The 1st International Forum on Strategic Technology, Ulsan, South Corea, IEEE.

Junli, W., D. Zhijun and J. Changjun (2006). GAOM: Genetic Algorithm based Ontology Matching. IEEE AsiaPacific Conference on Services Computing (APSCC'06), Guangzhou, Guangdong, China

Lehtoranta, S., A. Nissinen, T. Mattila and M. Melanen (2011). Industrial symbiosis and the policy instruments of sustainable consumption and production. Journal of Cleaner Production 19(1): pp $1865 \mathrm{e} 1875$.

Melnik, S., H. Garcia-Molina and E. Rahm (2002). Similarity flooding - a versatile graph matching algorithm. 18th International Conference on Data Engineering. San Jose, CA, USA: pp 182-200.

Mirata, M. and T. Emtairah (2005). Industrial symbiosis networks and the contribution to environmental innovation: The case of the Landskrona industrial symbiosis programme Journal of Cleaner Production 13(10-11): pp 993-1002.

Raafat, T., F. Cecelja, N. Trokanas and B. Xrisha (2013). An Ontological Approach Towards Enabling Processing Technologies Participation in Industrial Symbiosis. Computers \& Chemical Engineering 59(1): pp 33-46.

Tous, R. and J. Delgado (2006). A vector space model for semantic similarity calculation and OWL ontology alignment. 17th International Conference on Database and Expert Systems Applications, DEXA 2006. Kraków, Poland,: pp 307-316.

Trokanas, N., F. Cecelja and T. Raafat (2014). Semantic Input/Output Matching for Waste Processing in Industrial Symbiosis. Computers \& Chemical Engineering 66(1): pp 259 - 268.

Trokanas, N., F. Cecelja and T. Raafat (2015). Semantic Approach for Pre-assessment of Environmental Indicators in Industrial Symbiosis. Journal of Cleaner Production 96(1): pp 349-361.

Trokanas, N., F. Cecelja, T. Raafat and P. Innes (2013). An Ontological Approach Enabling "a Priori" Quantitative Assessment of Is Networks. 2013 AIChE Annual Meeting, San Francisco, USA.

Turban, E. and J. Aronson (2001). Decision Support Systems and Intelligent Systems. New Jersey, PrentinceHall, Inc. 